$\widehat{\underline{\underline{\underline{I I I I I I}}}}$

GHENT

UNIVERSITY
(") FACULTY

OF SCIENCES

Acta Materialia, Volume 183, 15 January 2020, Pages 493-503

\title{
Relating structural phase transitions to mechanoluminescence: The case of the $\mathrm{Ca}_{1-x} \mathrm{Sr}_{x} \mathrm{Al}_{2} \mathrm{Si}_{2} \mathrm{O}_{8}: 1 \% \mathrm{Eu}^{2+}, \mathrm{Pr}^{3+}$ anorthite
}

\author{
Ang Feng ${ }^{a, b}$, Simon Michels ${ }^{a, b}$, Alfredo Lambertic, Wim Van Paepegem ${ }^{c}$, Philippe F. Smet ${ }^{a, b,{ }^{*}}$
}

a LumiLab, Department of Solid State Sciences, Ghent University, Krijgslaan 281-S1, Gent 9000, Belgium

b Center for Nano and Biophotonics (NB-Photonics), Ghent University, Gent 9000, Belgium

c Mechanics of Materials and Structures (MMS), Department of Materials, Textiles and Chemical Engineering (MaTCh), Ghent University, Tech Lane Ghent Science Park-Campus Ardoyen 46, Zwijnaarde 9052, Belgium

* Corresponding author at: LumiLab, Department of Solid State Sciences, Ghent University, Krijgslaan 281-S1, Gent9000, Belgium.

E-mail address: philippe.smet@UGent.be (P. F. Smet)

This is the accepted version after peer review, and the publisher's version can be found via the DOI link below

https://doi.org/10.1016/j.actamat.2019.11.014

Graphical abstract

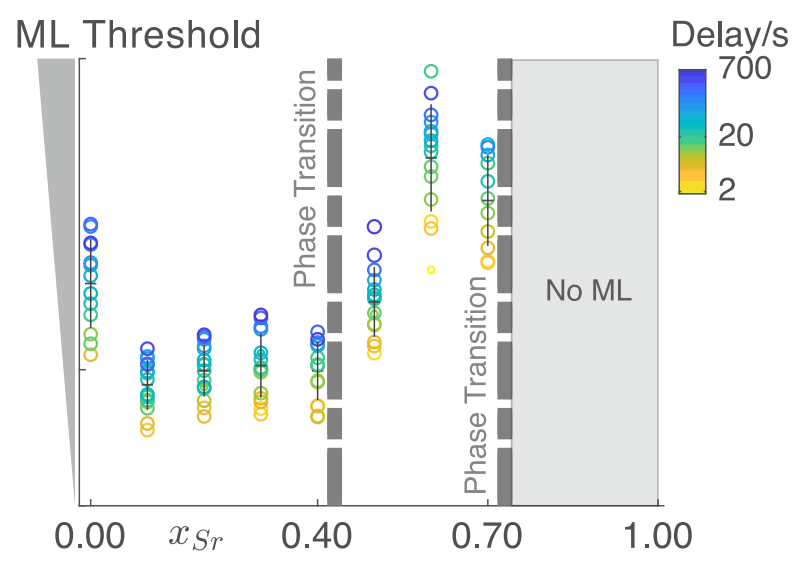

C 2019. This manuscript version is made available under the CC-BY-NC-ND 4.0 license

http://creativecommons.org/licenses/by-nc-nd/4.0/

(1) (1) $\Theta \Theta$ 


\title{
Relating Structural Phase Transitions to Mechanoluminescence: The Case of the $\mathrm{Ca}_{1-x} \mathrm{Sr}_{x} \mathrm{Al}_{2} \mathrm{Si}_{2} \mathrm{O}_{8}: 1 \% \mathrm{Eu}^{2+}, 1 \% \mathrm{Pr}^{3+}$ Anorthite
}

\author{
Ang Feng ${ }^{\mathrm{a}, \mathrm{b}}$, Simon Michels ${ }^{\mathrm{a}, \mathrm{b}}$, Alfredo Lamberti ${ }^{\mathrm{c}}$, Wim Van Paepegem ${ }^{\mathrm{c}}$, Philippe F. Smet ${ }^{\mathrm{a}, \mathrm{b}, *}$ \\ ${ }^{a}$ LumiLab, Department of Solid State Sciences, Ghent University, Krijgslaan 281-S1, 9000 Gent, Belgium \\ ${ }^{b}$ Center for Nano and Biophotonics (NB-Photonics), Ghent University, 9000 Gent, Belgium \\ ${ }^{c}$ Mechanics of Materials and Structures (MMS), Department of Materials, Textiles and Chemical Engineering (MaTCh), Ghent \\ University, Tech Lane Ghent Science Park-Campus Ardoyen 46, 9052 Zwijnaarde, Belgium
}

\begin{abstract}
The phenomenon of mechanoluminescence (ML), where phosphors emit light when pressure is applied, is considered to be closely related to the crystallographic structure of those phosphors. In this work we unravel this connection for the anorthite solid solution $\mathrm{Ca}_{1-x} \mathrm{Sr}_{x} \mathrm{Al}_{2} \mathrm{Si}_{2} \mathrm{O}_{8}$, which displays two important phase transitions as a function of strontium content $x$ (denoted as $x_{S r}$ ), i.e., the nearly second-order P $\overline{1}-\mathrm{I} \overline{1}$ transition and the ferroelastic I $\overline{1}-\mathrm{I} \frac{2}{c}$ transition at ambient temperature and pressure. The spontaneous strains reveal that the ferroelastic transition takes place when $x_{S r} \in(0.70,0.75)$, while other optical methods suggest that the second-order $\mathrm{P} \overline{1}-\mathrm{I} \overline{1}$ transition takes place when $x_{S r}$ is around 0.4. The ML intensity reaches its maximum when the second order transition takes place and drops to zero when the phosphors undergo the ferroelastic transition. The first transition already brings significant changes to electron occupations at traps in this solid solution. The structural phase transitions in the anorthite solid solutions are reflected in specific ML properties, such as the ML intensity and the load threshold. Further analysis suggests this is due to the structural change of the hosts and the trap properties (trap density and electron population function). Analysis of the ML dynamics may therefore serve as a useful tool to investigate phase transitions in ML phosphors.
\end{abstract}

Keywords: solid solution, phase transition, mechanoluminescence, thermoluminescence, defect

\section{Introduction}

Mechanoluminescence is the non-thermal emission of light due to mechanical stimulation applied to solid materials [1. ML in inorganic luminescent materials (or phosphors) is particularly interesting because of the underlying physics and the potential application as stress sensor [2], ML display [3, 4], wearable ML textile [5], smart skin [6], and information storage [7, 8]. It generates even extended and unexpected applications when coupled to other functionality of materials such as piezoelectricity [9], magnetostriction 10, triboelectricity [11, upconversion luminescence [8], etc. While a few ML phosphors are based on triboelectricity [12] or the movement of dislocations [13, many others, which are often persistent phosphors, are based on the accelerated detrapping of previously trapped charges (usually electrons) due to mechanical stimulation (see Ref 1, 14 for a review). Naturally, the ML intensity and kinetics depend on the trap depth distribution, the resultant electron population function and the trapping and detrapping mechanisms. Different populations of electrons in traps can be achieved by different charging conditions, such as charging duration, wavelength and temperature,

\footnotetext{
* Corresponding author

Email address: Philippe.Smet@UGent.be (Philippe F. Smet )
}

or by a delay between the end of charging and the start of the mechanical stimulation.

Usually, the fundamental properties of traps in ML phosphors vary from one host-dopant combination to another. Solid solutions serve as a valuable research tool due to their capability to induce continuous variation of structural properties between the involved end-member compounds. For example, ML in the $(\mathrm{Ba}, \mathrm{Ca}) \mathrm{TiO}_{3}: \mathrm{Pr}^{3+}$ system is enhanced by the increase of electro-restriction near the solution limits 15, 16. A reduction of trap density can enhance ML contrast, facilitating stress visualization [17]. Chemical substitution can also introduce possible phase transitions, among which second-order transitions exhibit interesting phenomena near the transition point. The correlation length increases to infinity there [18] and physical properties emerge that are absent in their end-member compounds, such as stiffening or softening of the structure [19], huge enhancement of piezoelectricity [20, 21], or anomalies of the specific heat capacity [22]. Structural phase transitions can be observed via these macroscopic quantities if the change of free energy $(\triangle G)$ is large enough, otherwise they are translated into more subtle changes, like atomic disorder, changes in textures or the occurrence of superstructures. In this context, ML acts as a means to probe the change of the elasticity of ML phosphors or/and their local structures and defects via lu- 
minescence, as a response to dynamic stress.

Anorthite belongs to the feldspar family, which is characterized by a framework of ordered $\left[\mathrm{AlO}_{4}\right] /\left[\mathrm{SiO}_{4}\right]$ tetrahedra with cations stuffed in the cavities [23]. The anorthite solid solution $\mathrm{Ca}_{1-x} \mathrm{Sr}_{x} \mathrm{Al}_{2} \mathrm{Si}_{2} \mathrm{O}_{8}$ is an ideal system to reveal the interaction of ML with structural phase transitions. At ambient temperature and pressure, it displays, as $x_{S r}$ increases, first a nearly second-order P $\overline{1}-\mathrm{I} \overline{1}$ transition, where the cation sites are splitted (atom-split) but the aluminosilicate framework stays unchanged. At even higher $x_{S r}$, another ferroelastic I1 $-\mathrm{I} \frac{2}{c}$ transition occurs, where the symmetry increases and the framework becomes disordered at the $\left[\mathrm{AlO}_{4}\right] /\left[\mathrm{SiO}_{4}\right]$ tetrahedra. At high temperature $(\sim 514 \mathrm{~K})$ or high strontium content, the calcium end-member $\mathrm{CaAl}_{2} \mathrm{Si}_{2} \mathrm{O}_{8}$ transforms to the $\mathrm{I} \overline{1}$ structure by an atom-split at $\mathrm{Ca}^{2+}$ sites, with the $\mathrm{I} \overline{1}$ structure being the dynamical average of small $\mathrm{P} \overline{1}$ domains 24. This transition involves $\triangle G$ that is too small to be observable by macroscopic properties like unit cell parameters, but it induces rich local structures and possible trapping levels within the band-gap. The strontium counterpart, $\mathrm{SrAl}_{2} \mathrm{Si}_{2} \mathrm{O}_{8}$, crystallizes in a similar structure $\left(\mathrm{I} \frac{2}{c}\right)$ with random $\mathrm{Al}^{3+} / \mathrm{Si}^{4+}$ occupation at tetrahedra sites and transforms to the $\mathrm{I} \overline{1}$ phase at elevated pressure [25] or via $\mathrm{Ca}^{2+}$ doping [26]. In the solid solution, the $\mathrm{I} \overline{1}$ phase differs from the calcium end-member $(\mathrm{P} \overline{1})$ by an atom-split of the cation sites, while it differentiates from the Sr end-member $\left(\mathrm{I} \frac{2}{c}\right)$ by the ordering of tetrahedra and symmetry-breaking of the framework structure. The effect of $\mathrm{Sr}^{2+}$ substitution is to increase the cation polyhedral volume, expanding the unit cell, and to excite two tilting modes of tetrahedra, which are responsible for the elasticity anisotropy that enables anorthites to behave like layered silicates [27]. Doping luminescent ions into anorthites may break the symmetry of the host, as is the case for some centrosymmetric niobate ML phosphors 28. Furthermore, the interplay between elastic anisotropy, local defects and luminescence may alter the relevant processes of ML, thus bringing new strategies to tune the properties of ML phosphors at large. Indeed, a variation of the ML intensity with the $\mathrm{Sr}^{2+}$ content in this solid solution was observed in $\mathrm{Eu}^{2+}$ singly doped [29] and $\mathrm{Eu}^{2+}-\mathrm{Dy}^{3+}$ codoped 30] phosphors, where ML intensity reached its peak at $x_{S r}=0.40$ and disappeared at $x_{S r}=0.80$. This suggests an interaction between ML and the chemical composition $x_{S r}$. In this contribution, we probed the phase transitions in anorthite $\mathrm{Ca}_{1-x} \mathrm{Sr}_{x} \mathrm{Al}_{2} \mathrm{Si}_{2} \mathrm{O}_{8}: 1 \% \mathrm{Eu}^{2+}, 1 \% \mathrm{Pr}^{3+}$ by $\mathrm{ML}$ and other methods that are both sensitive to the change of the aluminosilicate framework, such as cell parameters and photoluminescence (PL) spectra, and sensitive to local defects and the associated trap levels, such as internal quantum efficiency (IQE), afterglow, thermoluminescence (TL) and electron population function at traps. ML is found to be sensitive to both the elasticity of anorthites and their local structures and thus serves as an excellent means to study the phase transitions by ML properties such as ML peak intensity, ML threshold, and ML dy- namics. It enriches our understanding of ML and opens an additional toolbox for investigating phase transitions in ML phosphors.

\section{Experimental Details}

\subsection{Phosphor Synthesis}

$\mathrm{Ca}_{1-x} \mathrm{Sr}_{x} \mathrm{Al}_{2} \mathrm{Si}_{2} \mathrm{O}_{8}: 1 \% \mathrm{Eu}^{2+}, 1 \% \mathrm{Pr}^{3+}$ phosphors were prepared by a solid-state reaction method. Stoichiometric amounts of $\mathrm{CaCO}_{3}$ (Alpha Aesar, 99.99\%), $\mathrm{SrCO}_{3}$ (Alpha Aesar, 99.99\%), $\mathrm{SiO}_{2}$ (Alpha Aesar, 99.99\%, amorphous), $\mathrm{Eu}_{2} \mathrm{O}_{3}$ (Alpha Aesar, 99.99\%), $\mathrm{Al}_{2} \mathrm{O}_{3}$ (Fluka, 99.9\%, 10 $\mu \mathrm{m}$ ), and $\mathrm{Pr}_{6} \mathrm{O}_{11}$ (Alpha Aesar, 99.99\%) were weighted, mixed and ground in an agate mortar for $20 \mathrm{~min}$. The mixed powders were pressed into pellets (diameter $16 \mathrm{~mm}$, thickness 2-3 mm) at a pressure of approximately $100 \mathrm{MPa}$ for $3 \mathrm{~min}$. Subsequently, the pellets were placed into a corundum crucible and transferred to a water-cooled horizontal furnace, heated to $1350{ }^{\circ} \mathrm{C}$ at a rate of $5^{\circ} \mathrm{C} \cdot \mathrm{min}^{-1}$, maintained at that temperature for $6 \mathrm{~h}$, and cooled to 900 ${ }^{\circ} \mathrm{C}$ at a rate of $2.5^{\circ} \mathrm{C} \cdot \mathrm{min}^{-1}$. Finally, they were cooled down to room temperature naturally. A constant flow (rate 0.16 liter $\cdot \mathrm{min}^{-1}$ ) of $\mathrm{N}_{2} 95 \%-\mathrm{H}_{2} 5 \%$ forming gas was used in the entire process of heating and cooling. The solid pellets were crushed and ground for 30 min into fine powders before further characterization.

\subsection{X-Ray Diffraction}

All powder X-Ray diffraction (XRD) measurements were carried out on a Siemens D5000 diffractometer, which operated at $40 \mathrm{kV}$ and $40 \mathrm{~mA}$ with $\mathrm{Cu} \mathrm{K} \alpha$ radiation $(\lambda=$ $1.54215 \AA$ ) in the Bragg-Brentano geometry. The scanning range of $2 \theta$ was $10-120^{\circ}$ with a step-size of $0.02^{\circ}$ and the speed was $0.001^{\circ} \cdot \mathrm{sec}^{-1}$. The Rietveld refinement was carried out with the FullProf Suite 31] software.

\subsection{Optical Spectroscopy}

PL spectra were collected on an Edinburgh Instrument FLS920 spectrometer at room temperature. The wavelength step during the measurements was $0.25 \mathrm{~nm}$. All spurious spikes in the spectra were removed and then smoothed with the software SpectraGryph [32]. Afterglow spectra, together with the corresponding PL spectra, were measured with an EMCCD camera (Princeton Instruments ProEM ${ }^{\circledR}$ 1600) that was coupled to a spectrograph (Princeton Instruments Acton SP2300). Before taking spectra, the phosphors were charged for $2 \mathrm{~min}$ by UV light $(360 \mathrm{~nm})$ from a Xenon lamp combined with a fibercoupled grating monochromator. The same EMCCD camera and spectrograph, together with an integrating sphere (LabSphere GPS-SL series), were used to measure the IQE of anorthites upon light-emitting-diode (LED) excitation at $365 \mathrm{~nm}$ (full width at half maximum $(\mathrm{FWHM}) \sim 20$ $\mathrm{nm})$. 


\subsection{Afterglow Duration Test}

The afterglow (AG) duration was measured with a calibrated photometer (ILT 1700, International Light Technology) equipped with a photopic filter (YPM). Powders were weighted $(0.3 \mathrm{~g})$ and placed on an aluminum plate with a holder (diameter $16 \mathrm{~mm}$, depth $1 \mathrm{~mm}$ ), and illuminated by a Xenon lamp with 1000 lux for $10 \mathrm{~min}$. Then, the plate was transferred exactly underneath the photometer (at a distance of approximately $20 \mathrm{~mm}$ ) in a dark box and kept untouched until the luminescence fell below $2 \times 10^{-2}$ $\mathrm{mCd} \cdot \mathrm{m}^{-2}$. The afterglow duration is defined to be the time it takes for the afterglow to drop below the $0.32 \mathrm{mCd}$. $\mathrm{m}^{-2}$ threshold.

\subsection{Thermoluminescence and Thermal Quenching}

Phosphor powders were pressed into small discs (diameter of $5 \mathrm{~mm}$ ) and then placed into a home-built setup as described in Ref 33. A normal thermoluminescence curve can be acquired in the following way. After excitation with $370 \mathrm{~nm}$ light from a LED $(60 \mathrm{~mA})$ for $5 \mathrm{~min}$, the discs were cooled down to $-60{ }^{\circ} \mathrm{C}$ and then heated up to $225^{\circ} \mathrm{C}$ at a fixed rate of $0.5^{\circ} \mathrm{C} \cdot \mathrm{sec}^{-1}$. To reveal the electron population function, the $T_{m}-T_{\text {stop }}$ method was adopted according to Ref [34] (section 3 in the ESI). After the UV excitation, the phosphor was heated to $T_{\text {stop }}\left(\right.$ rate $0.5^{\circ} \mathrm{C} \cdot \mathrm{sec}^{-1}$ ) and then immediately cooled down to a low temperature $\sim-60{ }^{\circ} \mathrm{C}$ (rate $0.5^{\circ} \mathrm{C} \cdot \mathrm{sec}^{-1}$ ), and finally heated up to 225 ${ }^{\circ} \mathrm{C}$ (rate $\left.0.5^{\circ} \mathrm{C} \cdot \mathrm{sec}^{-1}\right)$. The luminescence was detected by the ILT1700 photometer. For thermal quenching measurements, the phosphors were illuminated by a $370 \mathrm{~nm}$ LED (60 $\mathrm{mA}$ ) at the charging temperature $T$ for $60 \mathrm{sec}$, while the spectra were acquired by using the EMCCD camera and spectrograph described in section 2.3. Four spectra corresponding to the time slot of $\sim 54-55$ sec of the charging duration were averaged to calculate the PL emission intensity. The charging temperature $T$ was increased from $-60{ }^{\circ} \mathrm{C}$ to $225^{\circ} \mathrm{C}$ in steps of $5^{\circ} \mathrm{C}$. After interpolating the relative $\mathrm{PL}$ intensity at each temperature by a cubic spline method, all TL profiles were corrected by the corresponding thermal quenching file.

\subsection{Profiles Measurement}

Coupon samples (length $>120 \mathrm{~mm}$, width $12.5 \mathrm{~mm}$, thickness $2.0 \mathrm{~mm}$ ) for ML measurements were prepared by the resin transfer molding method 35. Resin (Epikote ${ }^{T M}$ Resin MGS LR 135) and hardener (Epikote ${ }^{T M}$ Resin MGS LR 137) were taken in a 10:3 weight ratio and mixed with ML powders (weight ratio $3 \%$ ). They were degassed for 2 min to remove the air bubbles. By using a vacuum, the mixture was infused into a mold and cured at room temperature for $24 \mathrm{~h}$ before post-curing at $80{ }^{\circ} \mathrm{C}$ for 15 h. Tensile experiments were carried out with an Instron ${ }^{\circledR}$ ElectroPuls $^{T M}$ E10000 instrument, which offers accurate loading through either digital displacement or force. A normal ML profile can be obtained in the following way, as illustrated in Figure 1a. The stress-free coupon was charged with a UV lamp (6 W, centered at $365 \mathrm{~nm})$ for $150 \mathrm{sec}$ (Charging), then left at rest for a period (Delay) before an extension of $0.6 \mathrm{~mm}$ at a rate of $1.0 \mathrm{~mm} \cdot \mathrm{sec}^{-1}$, which was confirmed to remain in the linear range of deformation. The maximum deformation, i.e. $0.6 \mathrm{~mm}$, was attained for at least $280 \mathrm{sec}$ (Relaxation), before unloading to zero linearly in $30 \mathrm{sec}$. The light emission was collected by a Hamamatsu ${ }^{\circledR}$ H10722-210 multiplier tube (PMT), whose control voltage was set to $0.515 \mathrm{~V}$. A closed shutter was used to protect the PMT from strong illumination during charging, but it was opened manually when the charging ended (delay $\sim 0.1 \mathrm{sec}$ ), enabling the collection of luminescence. The voltage signal from the PMT was synchronized with voltage signals for digital displacement and force. All these signals were sampled at a rate of $2048 \mathrm{~Hz}$ via a National Instruments ${ }^{\circledR}$ data acquisition unit (NI9234), together with a CompactDAQ chassis (cDAQ9174).

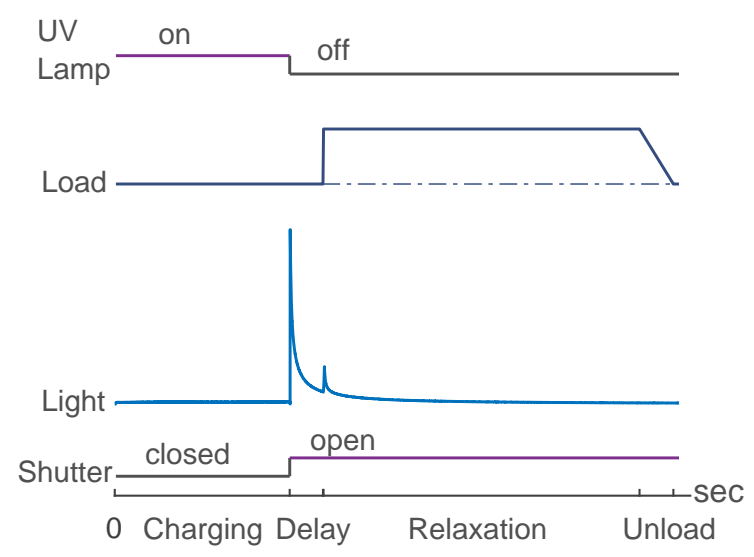

(a)

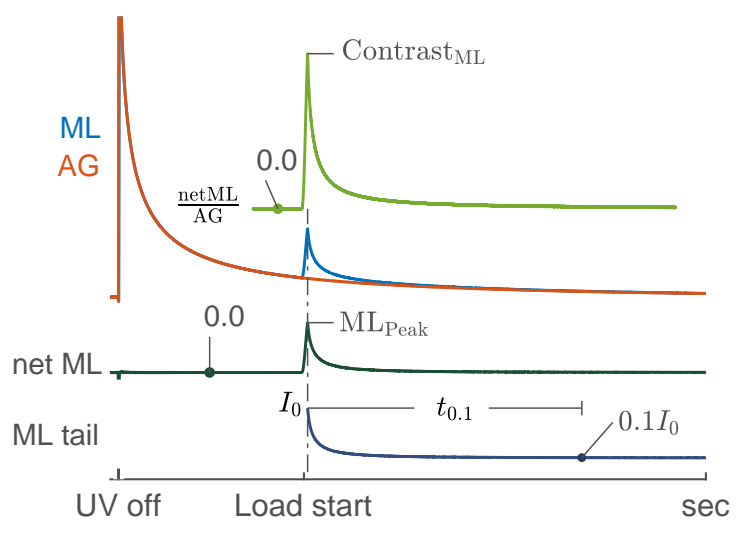

(b)

Figure 1: (a) The experimental procedures to study the ML properties include charging the phosphors with a UV lamp (centered at $365 \mathrm{~nm}$ ) for $150 \mathrm{sec}$, waiting some time (Delay) before applying the load, keeping the maximum load for $280 \mathrm{sec}$ (Relaxation) and unloading the sample (in $30 \mathrm{sec}$ ). (b) Information can be obtained by processing the ML profile (blue), together with the corresponding AG profile (red) in the absence of pressure. The net ML is simply ML-AG, whose maximum is $\mathrm{ML}_{\text {Peak }}$. The ML tail is the part of net ML after it passes $\mathrm{ML}_{\text {Peak }}$, and its maximum is denoted as $I_{0}$. The Contrast $_{\mathrm{ML}}$ is the maximum value of $\frac{\text { net } \mathrm{ML}}{\mathrm{AG}}$. 
Several types of information can be extracted from the ML profiles, as illustrated in Figure 1b. Subtracting the corresponding AG from a ML profile, one can define the net ML, which can be roughly reckoned as the luminescence due to the load without the contribution of AG. The maximum of the net ML is defined as $\mathrm{ML}_{\mathrm{Peak}}$, which is often obtained when the load reaches its maximum. The relative strength of net $\mathrm{ML}$ to $\mathrm{AG}$, i.e., $\frac{\text { net } \mathrm{ML}}{\mathrm{AG}}$, starts from zero, and changes with time when the load is applied. The maximum of $\frac{\text { net ML }}{\mathrm{AG}}$ is defined as Contrast ML $_{\text {. The part of }}$ net ML from the $\mathrm{ML}_{\text {Peak }}$ to the end of Relaxation is called ML tail, whose maximum is denoted by $I_{0}$. The duration for the ML tail to decay from its initial value $I_{0}$ to $0.1 I_{0}$ is denoted as $t_{0.1}$.

\section{Results and Discussion}

\subsection{The $I \overline{1}-I \frac{2}{c}$ Transition Takes Place at $x_{S r} \in(0.70,0.75)$}

The solid-state reaction method yields anorthites at the intended compositions. These phosphors are irregularlyshaped particles with diameters from $\sim 1 \mu \mathrm{m}$ to $\sim 30$ $\mu \mathrm{m}$ (Figure $\mathrm{S} 1$ in the electronic supplementary information (ESI)). Small amounts of $\alpha-\mathrm{Al}_{2} \mathrm{O}_{3}$ are present as impurities, as indicated in typical Rietveld refinement patterns and further confirmed by Energy-dispersive X-ray (EDX) measurements (Figure S2, Figure S3 and Table S1 in the ESI). The Rietveld refinements converged for all chemical compositions, reaching relatively small $\mathrm{R}$ values. For $\mathrm{CaAl}_{2} \mathrm{Si}_{2} \mathrm{O}_{8}$, one expects a phase transition from $\mathrm{P} \overline{1}$ to $\mathrm{I} \overline{1}$ at $514 \mathrm{~K}$ (ambient pressure), and the transition temperature $\mathrm{T}_{c}$ decreases with increasing strontium substitution, reaching room temperature when $x_{S r} \in(0.40,0.50)$ for $\mathrm{Ca}_{1-x} \mathrm{Sr}_{x} \mathrm{Al}_{2} \mathrm{Si}_{2} \mathrm{O}_{8}$ [24, 36. This leads to a natural choice of the $\mathrm{P} \overline{1}$ structural model for cell parameter calculations when $x_{S r} \leq 0.70$, since the $\mathrm{I} \overline{1}$ structure is related to $\mathrm{P} \overline{1}$ by an atom-split of the cation sites [23, 37, which can often be interpreted as statistical-dynamic average of the $\mathrm{P} \overline{1}$ structure 24].

The substitution of calcium with strontium brings the $\mathrm{I} \overline{1}-\mathrm{I} \frac{2}{c}$ phase transition at $x_{S r} \in(0.70,0.75)$ (Figure 2a 2b). All cell parameters experience a turn-around in this chemical range where the transition takes place (Table S2 in the ESI). In contrast, the $\mathrm{P} \overline{1}-\mathrm{I} \overline{1}$ transition can only be tentatively inferred to be at $x_{S r} \in(0.40,0.45)$ from the XRD patterns where the overlap of $(12 \overline{3})$ and $(1 \overline{3} 0)$ peaks is more pronounced in the $\mathrm{P} \overline{1}$ phase than that in the $\mathrm{I} \overline{1}$ phase (Figure 2b. Previous reports revealed that the I $\overline{1}-$ $\mathrm{I} \frac{2}{c}$ transition in $\mathrm{Ca}_{1-x} \mathrm{Sr}_{x} \mathrm{Al}_{2} \mathrm{Si}_{2} \mathrm{O}_{8}$ is mainly ferroelastic (second-order) in nature, with a deviation to triclinic transitions [26]. In the framework of mean-field theory, the order parameter $\mathrm{Q}_{o}$, active in $\mathrm{I} \frac{2}{c}$, is related to spontaneous strains in triclinic phases [26, 40],

$$
\begin{aligned}
& e_{4}=\frac{c \cdot \cos \alpha}{c_{o} \cdot \sin \beta_{o}^{*}}+\frac{\cos \beta_{o}^{*}}{\sin \beta_{o}^{*}} \cos \gamma \\
& e_{6}=\cos \gamma
\end{aligned}
$$

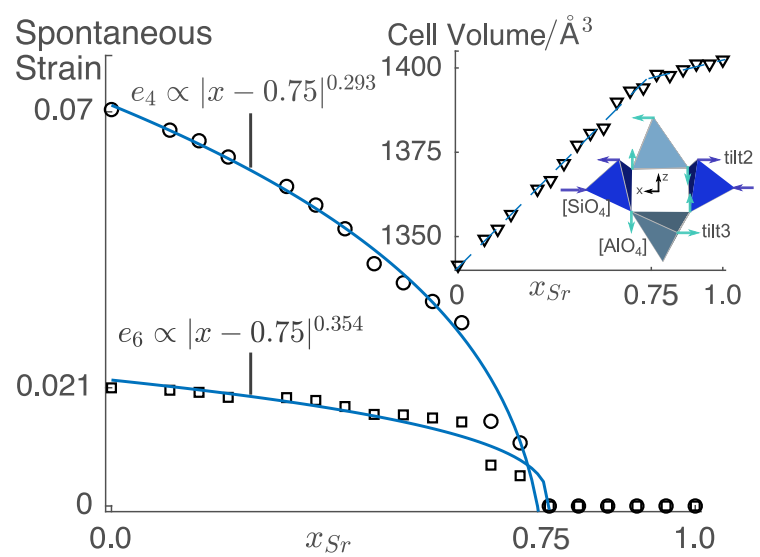

(a)

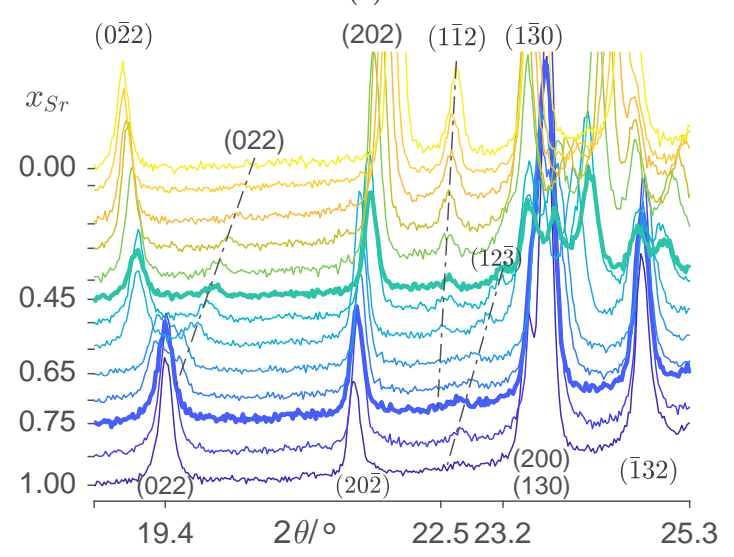

(b)

Figure 2: The spontaneous strains (a) $e_{4}$ and $e_{6}$ reduce to zero around $x_{S r} \simeq 0.75$, accompanied by a different change rate of cell volume and of the tilt angles of tetragedra (the inset, the image was re-drawn from Ref 38] with the VESTA software [39]). (b) The I $\overline{1}-$ $\mathrm{I} \frac{2}{c}$ structural transition can be inferred from this transition or the disappearance of $(0 \overline{2} 2)$ and $(1 \overline{1} 2)$ peaks of XRD patterns, while the $\mathrm{P} \overline{1}-\mathrm{I} \overline{1}$ transition can only be tentatively identified by the less overlap of $(12 \overline{3})$ and $(1 \overline{3} 0)$ peaks in the $\mathrm{I} \overline{1}$ phase than that in the $\mathrm{P} \overline{1}$ phase.

where the asterisk $*$ refers to the reciprocal space and the subscript $o$ to the paraelastic state (reference state). The corresponding parameters in the reference state can be calculated by extrapolation [40] (Table S2 and Figure S4 in the ESI). A clear drop of $e_{4}$ to zero near $x_{S r} \simeq 0.75$ (Figure 2a indicates the phase transition, although the critical point $x_{c}$ is somewhat lower than those reported elsewhere [26], i.e., $(0.86,0.91)$. This difference is probably due to the low sintering temperature and short sintering duration of the syntheses [41, as compared to those reported in literature, e.g. $1500{ }^{\circ} \mathrm{C}$ and $72 \mathrm{~h}$ [26]. The samples are very likely to have some disorder at the $\mathrm{Al}^{3+} / \mathrm{Si}^{4+}$ sites, which tends to drive the transition point to the $\mathrm{Ca}-$ rich end member [42, 43]. The critical exponent for $e_{4}$ $\left(e_{4} \sim Q_{o} \propto\left|x-x_{c}\right|^{\theta}\right), \theta$, is 0.293 , indicating that the phase transition is a combination of triclinic $\left(\theta=\frac{1}{4}\right)$ transition and a second-order $\left(\theta=\frac{1}{2}\right)$ one. The difference in scaling law between $e_{4}$ and $e_{6}$ with chemical composition also indicates the deviation from a simple second-order 
phase transition and this may be attributed to a secondorder parameter $Q_{s}$ that is coupled to $Q_{o}$ quadratically [26].

The increasing strontium content induces tilting of the tetrahedra but at a different rate before and after the phase transition at $x_{S r} \simeq 0.75$ (the inset of Figure 2a). The rigidity of $\left[\mathrm{AlO}_{4}\right]$ and $\left[\mathrm{SiO}_{4}\right]$ tetrahedra allows to characterize the structure of the anorthite with only four permitted tilting modes of tetrahedra 27. Here, the disorder of $\mathrm{Al}^{3+} / \mathrm{Si}^{4+}$ only plays a minor role, while the incorporation of larger cations, such as $\mathrm{Sr}^{2+}$, has a larger impact on the framework. Only two of these tilt systems, i.e., tilt2 and tilt3 (the inset of Figure 2a), characterized by an increase in tilt angles, are indicative of the expansion of cell volume due to chemical substitution. Thus, the tilts increase at a slower rate above the critical point than below the critical point, meaning the $\mathrm{I} \frac{2}{c}$ phase is the stiffest phase in the solid solution.

\subsection{The PL Spectra Reflect a Transition at $x_{S r} \simeq 0.70$}

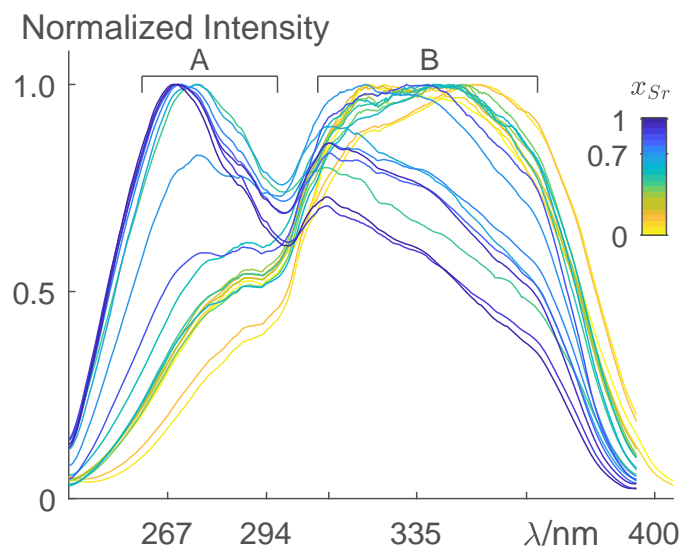

(a)

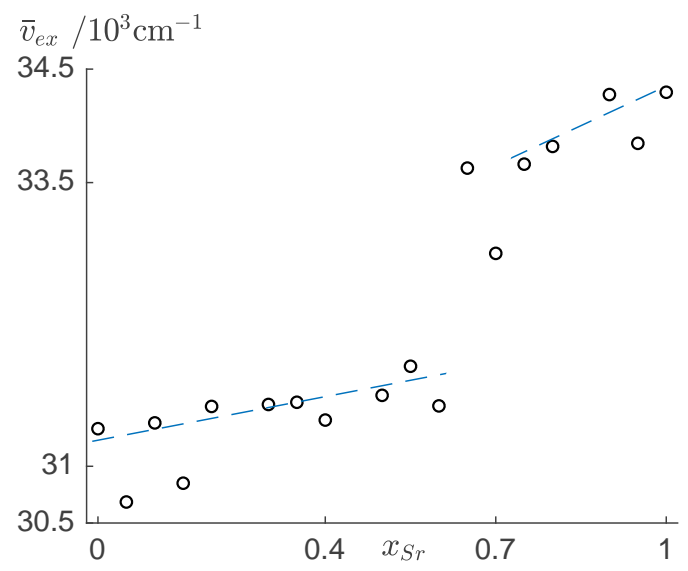

(b)

Figure 3: (a) Normalized PL excitation spectra of $\mathrm{Ca}_{1-x} \mathrm{Sr}_{x} \mathrm{Al}_{2} \mathrm{Si}_{2} \mathrm{O}_{8}$, display two bands, i.e., band A (260-295 $\mathrm{nm})$, and band B (300-360 nm), and (b) the effective excitation wave number shows a transformation at $x_{S r} \simeq 0.70$. The blue dashed lines serve as guides for the eyes.
Analysis of the PL spectra can reveal structural changes in anorthites, as the electronic $4 f^{6} 5 d^{1}-4 f^{7}$ transitions in $\mathrm{Eu}^{2+}$ are dependent on the local coordination. For all $x_{S r}$, PL excitation spectra (Figure 3a) display two excitation bands, one peaking at 260-290 nm (band A), and the other one at $310-350 \mathrm{~nm}$ (band B). The position and relative intensity of these bands evolve with $x_{S r}$, and the effective excitation wave number,

$$
\bar{\nu}_{e x}=\frac{\int I\left(\nu_{e x}\right) \nu_{e x} d \nu_{e x}}{\int I\left(\nu_{e x}\right) d \nu_{e x}}
$$

transforms at $x_{S r} \simeq 0.70$ (Figure 3b). The corresponding emission spectra (Figure 4a) consist of asymmetric bands centered at 405 to $429 \mathrm{~nm}$ due to the $4 f^{6} 5 d^{1}-4 f^{7}$ transition of $\mathrm{Eu}^{2+}$. As the $x_{S r}$ increases, the wavelength at

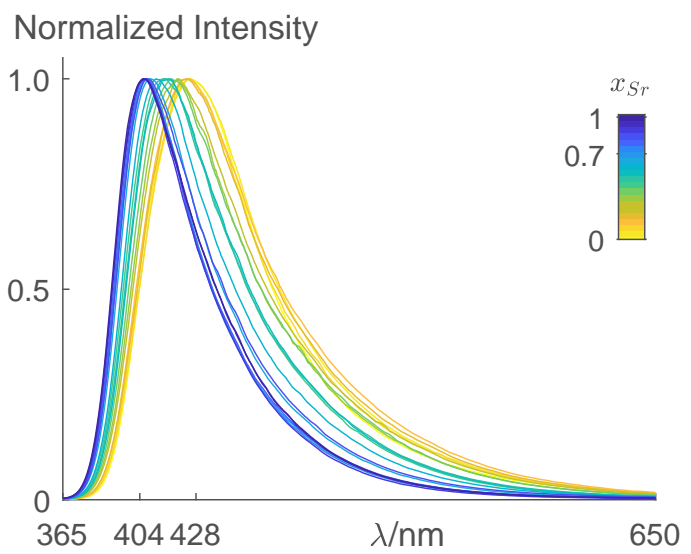

(a)

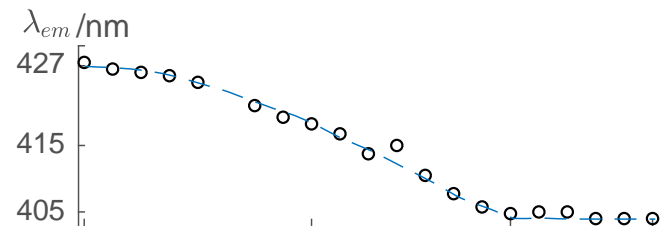

$\mathrm{FWHM} / \mathrm{nm}$

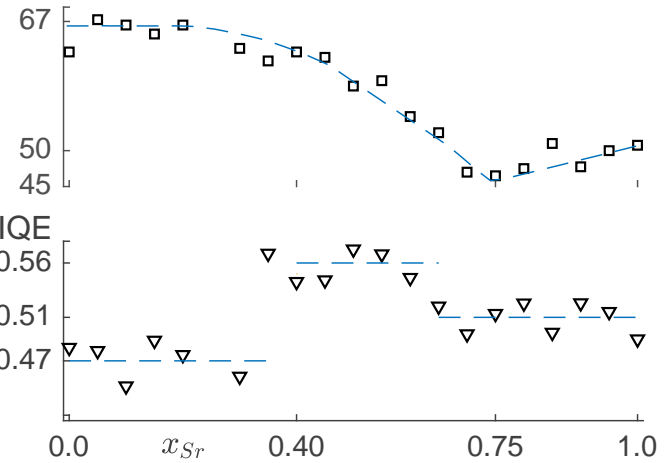

(b)

Figure 4: (a) The emission spectra of $\mathrm{Ca}_{1-x} \mathrm{Sr}_{x} \mathrm{Al}_{2} \mathrm{Si}_{2} \mathrm{O}_{8}$ are asymmetric bands. (b) The wavelength at emission maximum $\lambda_{e m}$ (top) and the corresponding FWHM (middle) decrease with increasing $x_{S r}$ before a transition point at $x_{S r} \simeq 0.75$. The IQE (bottom) upon $\lambda_{e x}=365 \mathrm{~nm}$ excitation indicates transitions at $x_{S r} \simeq 0.40$ and 0.70 . The blue lines serve as guides for the eyes. 
the emission maximum, i.e., $\lambda_{e m}$, decreases nonlinearly till $x_{S r} \simeq 0.70$ before stabilizing, while the corresponding FWHM increases slightly in the $(0.7,1.0)$ range after a similar nonlinear decrease in the range of $(0.0,0.70)$ (Figure 4b. The IQE upon $365 \mathrm{~nm}$ excitation (Figure $4 \mathrm{~b}$. bottom) shows two transitions at $x_{S r} \simeq 0.40$ and 0.70 , corresponding to the onset of the P $\overline{1}-\mathrm{I} \overline{1}$ and $\mathrm{I} \overline{1}-\mathrm{I} \frac{2}{c}$ transitions, respectively (see section 3.1). Such nonlinear behavior can only be revealed by densely sampling $x_{S r}$ in the anorthite solid solutions, otherwise false conclusions may be obtained, e.g. in Ref [4].

Clearly, the revealed transition behavior of PL spectra is related to the structural phase transition and the associated changes in the chemical environment of dopants. Compared with $\mathrm{Ca}^{2+}, \mathrm{Sr}^{2+}$ has a larger ionic radius [45] and smaller electronegativity [46], leading to smaller centroid shifts [47] with increasing $x_{S r}$. Besides, the magnitude of crystal field splitting is roughly reciprocal to the square of the averaged center-ligand distance of a cation polyhedron 48, 49, 50. Therefore, a blue shift of $\lambda_{\text {em }}$ of the emission band is expected before $x_{S r} \simeq 0.75$, after which $\lambda_{e m}$ stabilizes probably because of the high symmetry of the $\mathrm{I} \frac{2}{c}$ phase. Similarly, the average excitation wavenumber $\bar{\nu}_{e x}$ and the FWHM also evolve with increasing $x_{S r}$ and transform at $x_{S r} \simeq 0.70$ due to the ferroelastic transition. For a luminescent center, we have IQE $=$ $\frac{1}{1+\mathrm{k}_{\mathrm{nrad}} / \mathrm{k}_{\mathrm{rad}}}$ where $k_{\text {rad }}$ and $k_{n \text { rad }}$ are the coefficients of the radiative transition and the non-radiative transition, respectively. A high IQE value can be achieved by increasing the rigidity of the crystal structure to increases $k_{r a d}$ or/and by lowering the density of non-radiative centers to decrease $k_{n r a d}$. The disorders in the I $\overline{1}$ phase and the $\mathrm{I} \frac{2}{c}$ phase probably provide optimal bond of cation-anion or preferential occupation of $\mathrm{Sr}^{2+} / \mathrm{Eu}^{2+}$, as is the case of $(\mathrm{Ca}, \mathrm{Ba})_{2} \mathrm{SiO}_{4}: \mathrm{Eu}^{2+}$ [51, and thus a more rigid local structure enhances IQE. Furthermore, the IQE in a persistent phosphor depends on the trap density and the charging prcocess, such as the excitation intensity 52. The smaller the trap density (section 3.5), the higher the IQE value. Given a fixed trap density, a high ratio of shallow trap density to deep trap density (see section 3.5) effectively reduces trapping of electrons at traps, increasing the IQE value. Therefore, the IQE of the $\mathrm{I} \overline{1}$ phase and the $\mathrm{I} \frac{2}{c}$ phase, i.e., when $x_{S r} \geq 0.45$, are higher than that of the $\mathrm{P} \overline{1}$ phase of the anorthite solid solution.

\subsection{The Afterglow (AG) Reveals the Ferroelastic Phase Transition}

The afterglow results from the detrapping of electrons that populate traps according to the electron population function $n\left(E_{\text {trap }}, t\right)$ with $E_{\text {trap }}$ being the trap depth. Afterglow is observed at room temperature for the entire solid solution range for $x_{S r} \in(0.0,1.0)$, while their spectra agree well with their corresponding PL spectra when $x_{S r}<0.40$ but show additional emission at 470-600 nm when $x_{S r} \geq 0.40$ (Figure 5a). This is probably due to the distortion of cation sites, rendering possible preferential occupation of $\mathrm{Eu}^{2+}$ centers and energy transfer between them [53]. The distortion is severe when either the cation sites are partially occupied or the $\mathrm{Al}^{3+} / \mathrm{Si}^{4+}$ sites are disordered (randomly occupied), i.e., when $x_{S r} \geq 0.4$.

The afterglow profiles obey Becquerel's empirical decay law 54] (Figure 5b),

$$
I(t)=I_{0} \cdot(1+b \cdot t)^{-\beta} .
$$

The power factor $\beta$ is almost constant as $x_{S r}$ varies, yielding a mean value of 0.96 (the inset of Figure 5b). The afterglow duration (AG duration) is defined as the time needed for the afterglow to reach the threshold of 0.32 $\mathrm{mCd} \cdot \mathrm{m}^{-2}$ (the black horizontal line in Figure $5 \mathrm{~b}$ ). A careful analysis reveals that the AG duration is proportional to the total amount of afterglow intensity found by integrating $\mathrm{AG}$ to $2 \times 10^{-2} \mathrm{mCd} \cdot \mathrm{m}^{-2}$ (the inset of Figure $5 \mathrm{c}$, and thus it serves as a measure of afterglow. The AG duration as a function of $x_{S r}$ displays two straight lines in a $\log (\mathrm{AG}$ duration $)-x_{S r}$ plot, showing a turning point around $x_{S r} \simeq 0.65$, where the $\mathrm{I} \overline{1}-\mathrm{I} \frac{2}{c}$ phase transition takes place (Figure 5c).

Normally, it is quite hard to derive the mechanism of afterglow only from the decay law since it depends on numerous assumptions and the associated mathematical techniques. The decay function of afterglow can be regarded as an isothermal release of electrons in traps of previously charged phosphors [55]. Consider a phosphor with one kind of emitting center (density $m(t)$ ) and one kind of filled trap (density $n(t)=\int_{0}^{\infty} n\left(E_{\text {trap }}, t\right) d E_{\text {trap }}$ ) where recombination of electrons with emitting centers must happen via the conduction band (electron density $\left.n_{c}(t)\right)$. There might be fully filled thermally disconnected deep traps (density $C$ ) that do not participate in the luminescence processes. The afterglow profile is given by [56]

$$
I(t)=-\frac{d m}{d t}=\frac{m}{m+\gamma \cdot\left(N_{0}-n(t)\right)} \cdot \varphi(t)
$$

where $N_{0}$ is the total density of traps, and $\gamma=\frac{\sigma_{\text {retrap }}}{\sigma_{\text {recomb }}}$ the ratio of the cross section of retrapping $\left(\sigma_{\text {retrap }}\right)$ to the cross section of recombination $\left(\sigma_{\text {recomb }}\right) . \sigma_{\text {retrap }}$ is assumed to be independent of $E_{\text {trap }}$. Charge neutrality requires that $m(t)=n(t)+n_{c}(t)+C$. The function $\varphi(t)$ is actually an integration of first-order detrapping processes for electrons in the traps, i.e.,

$$
\varphi(t)=\int_{0}^{\infty} p\left(E_{\text {trap }}\right) n\left(E_{\text {trap }}, t\right) d E_{\text {trap }}
$$

where $p(E)=s \cdot \exp \left(-\frac{E_{\text {trap }}}{k_{B} T}\right)$ is the detrapping rate coefficient with $s$ being the 'attempt-to-escape' frequency, $k_{B}$ the Boltzmann's constant and $T$ the absolute temperature. Any afterglow function is $\varphi(t)$ corrected by a timedependent factor $\left(\frac{m}{m+\gamma \cdot\left(N_{0}-n(t)\right)}\right)$, leading to a possibly complicated function. Assuming the traps share only one trap depth, Adirovitch [57] achieved a solution of Equation 


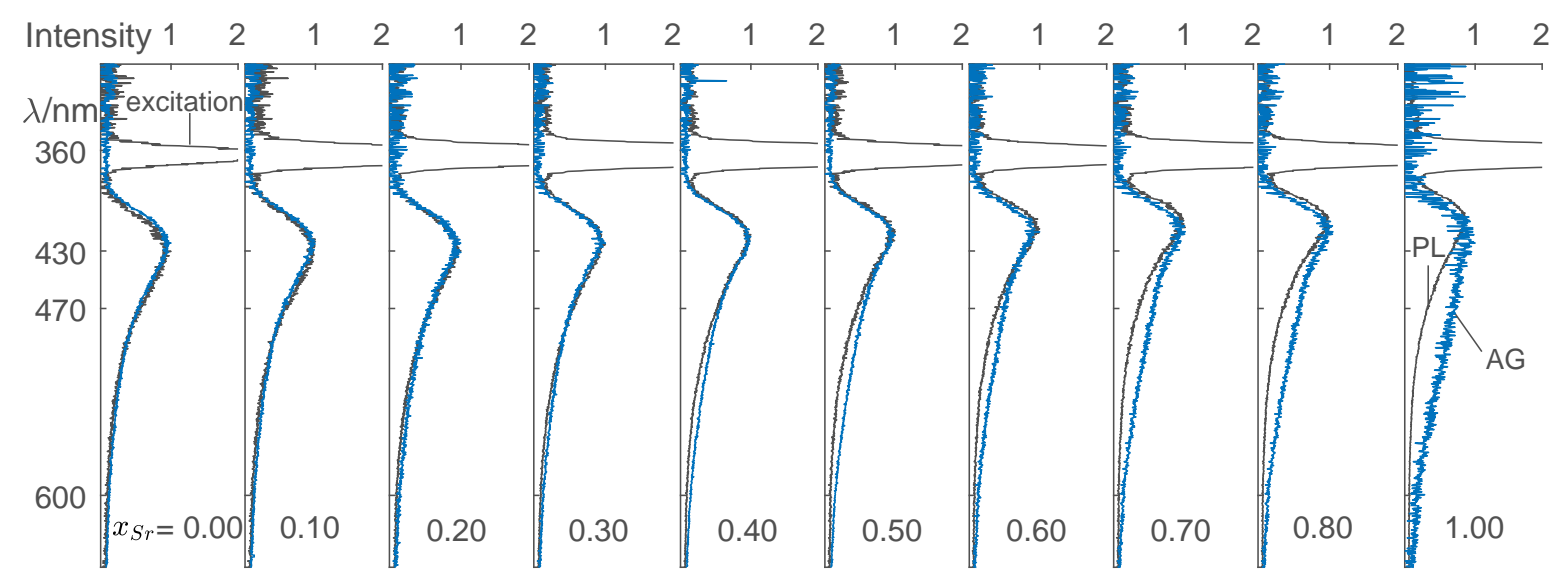

(a)

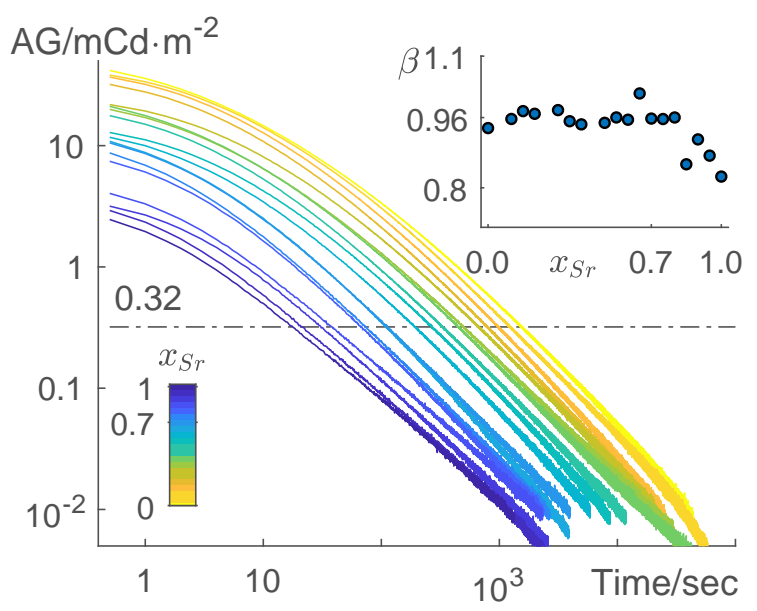

(b)

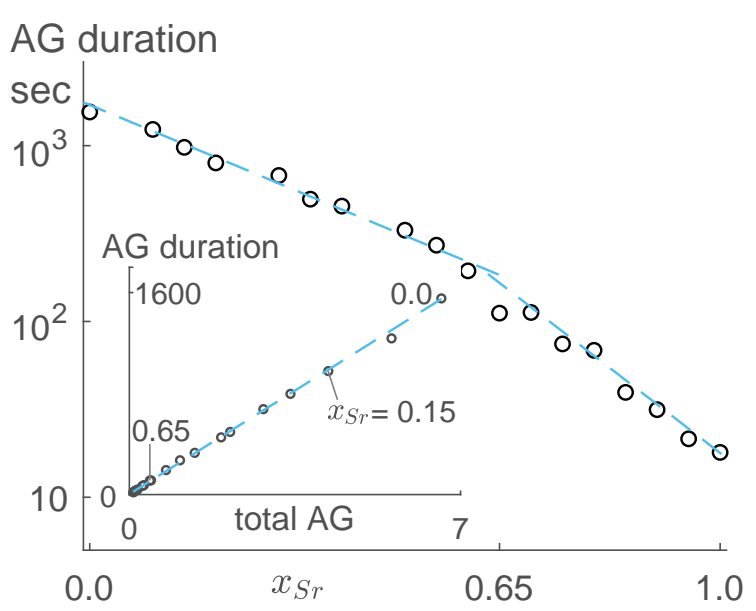

(c)

Figure 5: The AG spectra (blue, (a)) agree with the PL spectra (grey, (a)) when $x_{S r}<0.40$, but differ from PL spectra by additional emission at 470-600 nm when $x_{S r} \geq 0.40$. The afterglow profiles follow Becquerel's decay law (b), and the exponents $\beta$ vary slightly around 0.96 (the inset). The black horizontal line defines the AG threshold $0.32 \mathrm{mCd} \cdot \mathrm{m}^{-2}$. The AG duration (c), which is proportional to the corresponding total AG (the inset), decreases with increasing $x_{S r}$ by a power law, and shows a transition around $x_{S r} \simeq 0.65$. The blue dashed lines serve as guides for the eyes.

(4) by taking $m(t) \approx n(t)\left(C=0\right.$, and $\left.n_{c} \ll n\right)$, which yields,

$$
p\left(E_{\text {trap }}\right) t=(1-\gamma) \cdot \log \left(\frac{n_{0}}{n(t)}\right)-\gamma N_{0} \cdot\left(\frac{1}{n(t)}-\frac{1}{n_{0}}\right) .
$$

A map of $\gamma$ to $\beta$ in Becquerel's decay law (Equation (3p) was constructed by numerical calculation. The value of $\beta=0.96$ means $\gamma \sim 100$, which is 100 times higher than that of a second-order kinetics $(\gamma=1)$. This does not provide a physical explanation of the afterglow decay. A second order process does not produce the right asymptotic decay law, whether the traps are considered as a distribution or not [58]. The presence of thermally disconnected deep traps in real materials results in the prevalence of near first order kinetics of thermoluminescence [59. Note that the afterglow profiles for all the $x_{S r}$ follow the $t^{-1}$ behavior asymptotically, which is a characteristic of a distribution of decay rate coefficients if first-order kinetics are assumed [60, 61. Actually, the Becquerel decay law with $\beta \sim 1$ can result from a distribution of rate coefficients which is broad enough (for example Gaussian 62, exponential 63, Gamma 64, or even a uniform 63] distribution). Furthermore, the electron population function $n\left(E_{\text {trap }}, t\right)$ can be evaluated from the distribution $H(p)$ of the rate coefficient $p$ for first-order afterglow. Therefore, the AG profiles of the anorthite solid solutions reveal relatively broad probability distributions of the rate coefficients, and thus broad distributions of $n\left(E_{\text {trap }}, t\right)$ (section 3.5). However, the characteristics of $n\left(E_{\text {trap }}, t\right)$ were blurred inevitably in AG profiles dues to the integrating operation in $\varphi(t)$. This intrinsic property hinders AG from being a sensitive probe for traps in phosphors, especially for the electron population function.

\subsection{Properties Are Sensitive to Both Phase Transi- tions in the Anorthite Solid Solutions}

While the afterglow is determined by the electron population function and the thermally driven detrapping $\varphi(t)$, 
ML is initiated by the application of pressure. Both phenomena are closely related, as for instance, the application of pressure shortly after ending the excitation will superpose the ML response on the afterglow decay profile. On the other hand, the mechanically driven detrapping also induces some retrapping at shallow traps, leading to a quickly decaying emission intensity after ending the application of pressure, the so-called ML tail.

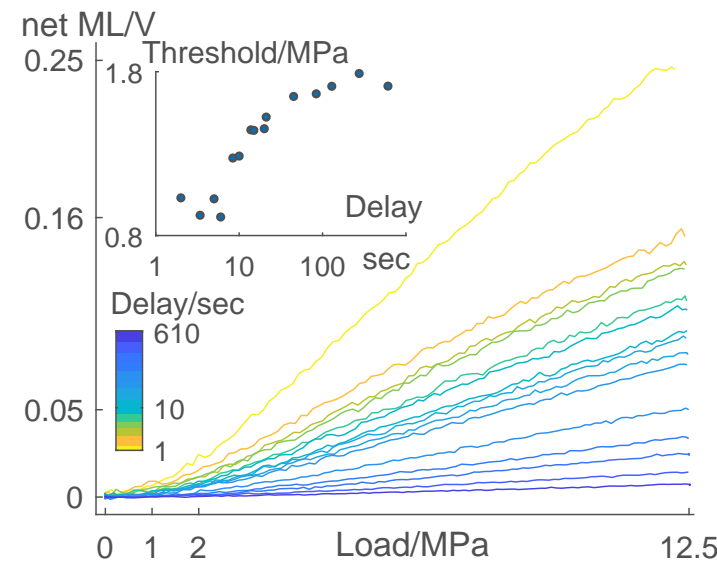

(a)

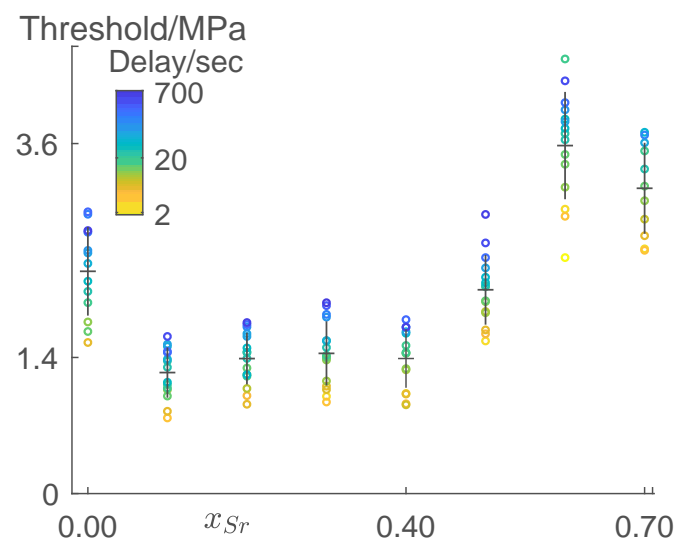

(b)

Figure 6: (a) For $x_{S r}=0.4$, the net ML increases linearly with load, after a threshold which increases with increasing Delay time investigated (the inset). (b) The threshold of net ML for all $x_{S r} \in$ $(0.0,0.70)$ is an increasing function of their corresponding Delay time. The average threshold (indicated by the horizontal lines) increases with increasing $x_{S r}$ for $x_{S r} \in(0.4,0.70)$. The vertical lines are the corresponding standard uncertainty.

The net ML responds linearly to the dynamic load after a threshold, which is dependent on the Delay time and also on the chemical composition $x_{S r}$. For example, the net ML for the $x_{S r}=0.40$ phosphor (Figure 6a) shows excellent linearity between the net ML and the load for all Delay ranging from 2 to $610 \mathrm{sec}$, after a threshold, ranging from 0.8 to $1.8 \mathrm{MPa}$ (the inset of Figure 6a). The threshold of ML for any $x_{S r} \leq 0.7$ in anorthite, as shown in Figure $6 \mathrm{~b}$, increases as the Delay time increases. Meanwhile, the average threshold (indicated by the horizontal lines) stays quite stable when $x_{S r} \leq 0.40$, and increases with $x_{S r}$ for higher strontium content. This suggests a transition in the relation between the threshold and $x_{S r}$ around $x_{S r}=0.4$, which coincides with the range where the $\mathrm{P} \overline{1}-\mathrm{I} \overline{1}$ transition takes place 65.

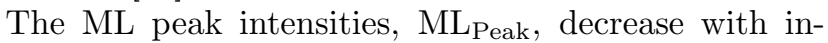
creasing Delay time (Figure 7a), following Becquerel's decay law (Equation (3) with $\beta$ values around 0.78 (the inset of Figure 7a), which is nearly independent of $x_{S r}$ in the solid solution. The ML disappears when $x_{S r}>0.70$, where the ferroelectric structural phase transition takes place (see Figure 2a. We introduce $\frac{1}{t_{0.1}}$ as the average decay rate function, where $t_{0.1}$ is the duration that the intensity of the ML tail takes to decay from $I_{0}$ to $0.1 I_{0}$. Clearly, $\frac{1}{t_{0.1}}$ also follows Becquerel's decay law with Delay, with $\beta$ values around 1.0 (Figure $7 \mathrm{~b}$ ). It is noteworthy that the fitting curves for $x_{S r} \in(0.0,0.4)$ are very close to each other but are very distinct from those for $x_{S r} \in(0.5,0.7)$. These two ranges set the ranges for the $\mathrm{P} \overline{1}-\mathrm{I} \overline{1}$ transition which was not revealed clearly in the structural studies. Furthermore, the $\beta$ values for $x_{S r}=0.7$ are high in both figures. This might be due to the closeness of the chemical composition to the critical transition point $x_{c}$, which is in the range $(0.7,0.75)$.

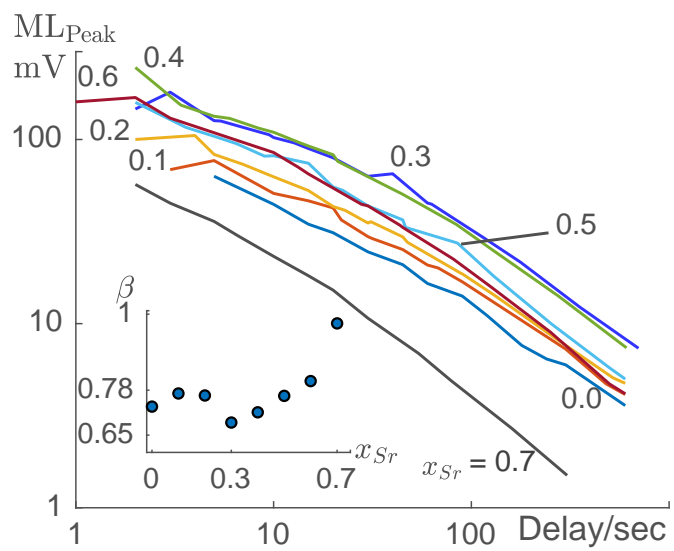

(a)

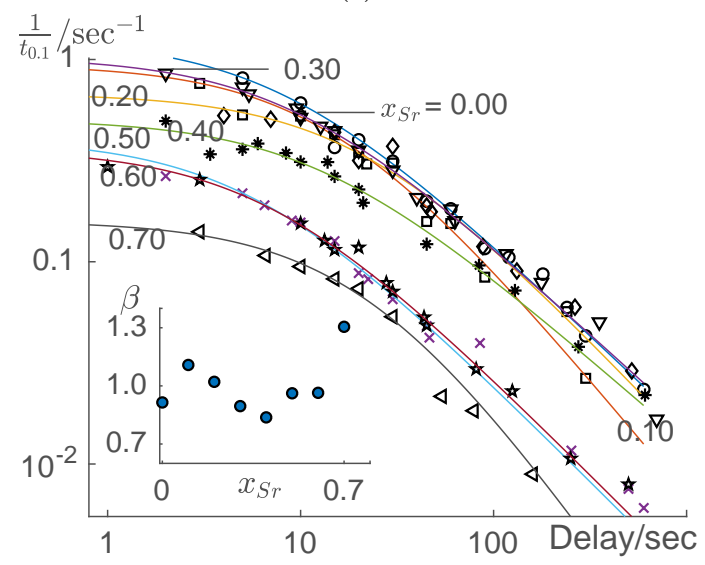

(b)

Figure 7: The $\mathrm{ML}_{\text {Peak }}$ (a) and the average transition rate $\frac{1}{t_{0.1}}$ (b) follow Becquerel's decay law with Delay time, while their $\beta$ values (in the corresponding inset) slightly depend on chemical composition, with mean values 0.78 and 1.0 , respectively. 
The Contrast of ML, Contrast $\mathrm{ML}=\max \left(\frac{\text { netML }}{\mathrm{AG}}\right)$, improves with increasing Delay time in a power law fashion and can be fitted by a straight line in a $\log \left(\right.$ Contrast $\left._{\mathrm{ML}}\right)$ $\log$ (Delay) plot, as shown in Figure 8 Since ML acts as an enhanced luminescence on top of $\mathrm{AG}$, Contrast $\mathrm{ML}$ is important to the understanding of ML dynamics and also for the visualization of stress distributions (see Ref [17] for example). Contrast ${ }_{M L}$ is a function of $x_{S r}$, and reaches its maximum when $x_{S r}=0.60$ for all the Delay times investigated. Interestingly, the Contrast $_{\mathrm{ML}}$ of $x_{S r}=0.40$ takes the second place, because of the strong $\mathrm{ML}_{\text {Peak }}$ and relatively low AG. These results suggest another way of increasing Contrast $_{\mathrm{ML}}$ due to the increase of ML in case of chemical substitution. This is quite different from the results of Zhang et al. 17, where both AG and ML decrease with increasing $\mathrm{Sr}^{2+}$ in $\left(\mathrm{Ca}_{1-x} \mathrm{Sr}_{x}\right)_{2} \mathrm{Nb}_{2} \mathrm{O}_{7}: \mathrm{Pr}^{3+}$, resulting in an improvement of Contrast ML due to the reduction of trap density.

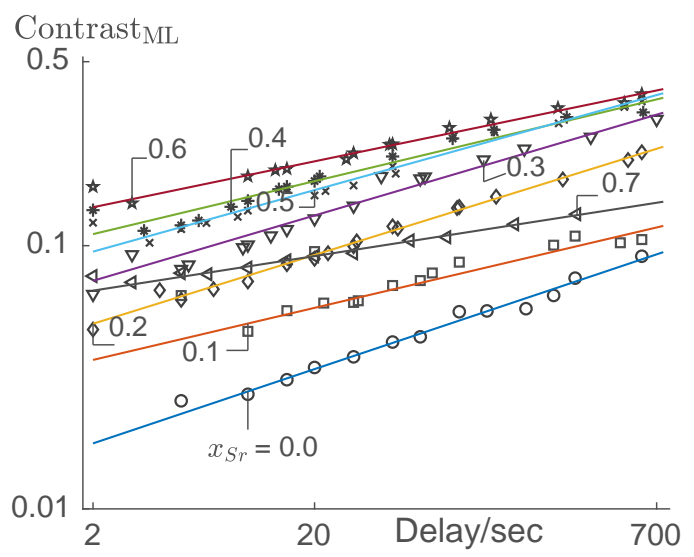

Figure 8: The Contrast ${ }_{\mathrm{ML}}$ (data: scattered points, fitting: lines) increases with Delay for all $x_{S r}$ in $\mathrm{Ca}_{1-x} \mathrm{Sr}_{x} \mathrm{Al}_{2} \mathrm{Si}_{2} \mathrm{O}_{8}$, following a power law. It is also a function of $x_{S r}$, reaching the maximum when $x_{S r}=0.6$ for all the Delay investigated.

It is possible to understand the ML characteristics (intensities, thresholds and tail kinetics) on the basis of the detrapping of the electrons from the traps. The amount of released electrons due to mechanical stimulation depends on the electron population function, and thus on the distribution of $p$ when first order kinetics are assumed. Through inverse Laplace transformation, Becquerel's decay law produces a Gamma distribution of rate coefficients 66, which leads to the corresponding time-dependent rate coefficient 67,

$$
k(t)=\frac{1}{\tau_{0}+t / \beta}
$$

where $\tau_{0}$ is the inverse of the "ensemble" average of $k$, i.e., $\tau_{0}=\frac{1}{\langle k\rangle}$. Clearly, $k(t)$ follows an inverse power law at large time limit. A different Delay time is actually probing the electron population function $n\left(E_{\text {trap }}, t\right)$, which is a monotonously decreasing function of $t$, at a different time. Thus, the ML $\mathrm{L}_{\text {Peak }}$ also decays with increased Delay time. As the Delay time increases, the electron population $n\left(E_{\text {trap }}, t\right)$ drops and a higher threshold for ML is expected and observed, assuming the conversion of dynamic load to detrapped electrons only depends on the phosphor itself. The evolution of average threshold with $x_{S r}$ clearly suggests that full ordering at both cation and tetrahedral sites is favorable for small thresholds, while partial occupation of cation sites often leads to increased threshold. Furthermore, the quantity $\frac{1}{t_{0: 1}}$ also arises from the accelerated detrapping rate which is related to $k(t)$, indicating a power law decay with $\beta \simeq 1$.

\subsection{Electron Population Functions Evolve with $x_{S r}$}

The electron population function $n\left(E_{\text {trap }}, t\right)$, which shows how electrons populate traps of different trap depth, is sensitive to the nature of traps in phosphors. It determines the magnitude and decay profile of the afterglow, given the value of $\gamma$. In addition, it can even predict the kinetics of ML if the mechanically stimulated detrapping of electrons is already known.

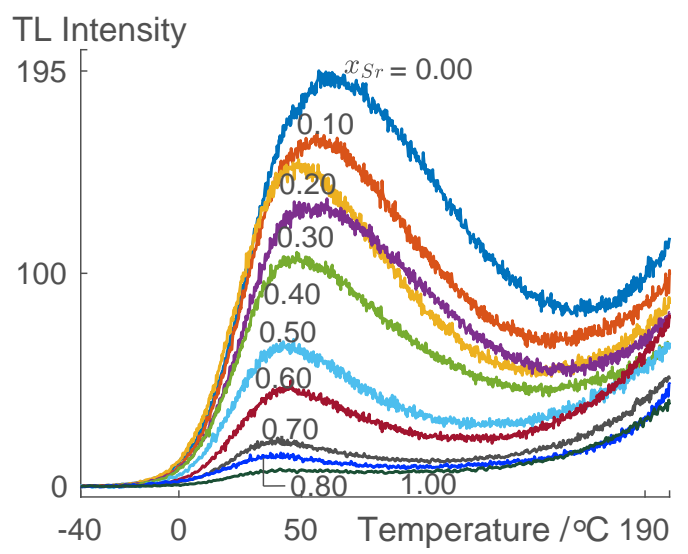

(a)

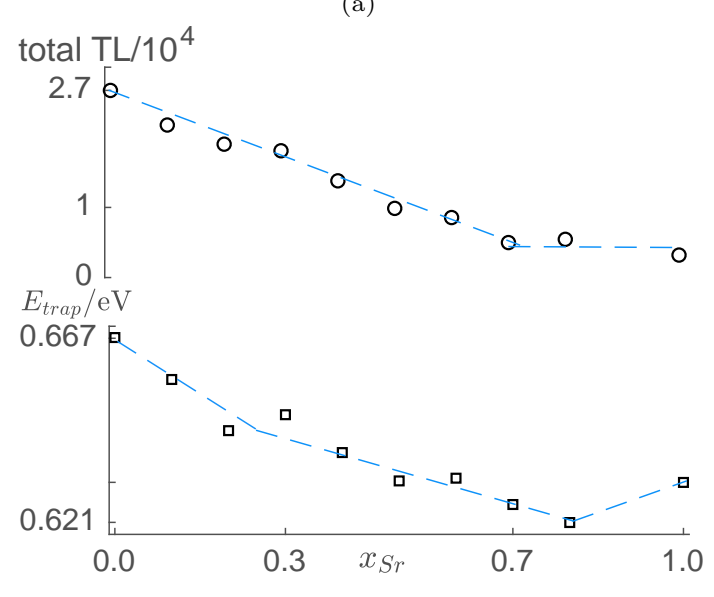

(b)

Figure 9: The TL curves (a) of the anorthite series after charging at $20^{\circ} \mathrm{C}$, and the corresponding total TL intensity (integration to $190{ }^{\circ} \mathrm{C}$ ) (b, top panel) decrease with increasing $x_{S r}$ while the trap depth (b, bottom panel) $E_{\text {trap }}$ decreases first to $x_{S r}=0.7$ and then increases slightly when $x_{S r} \in(0.7,1.0)$, indicating the transition point. Blue dashed lines serve as guides to the eyes.

The evolution in absolute magnitude of the afterglow intensity is in accordance with the trend of the TL peak 


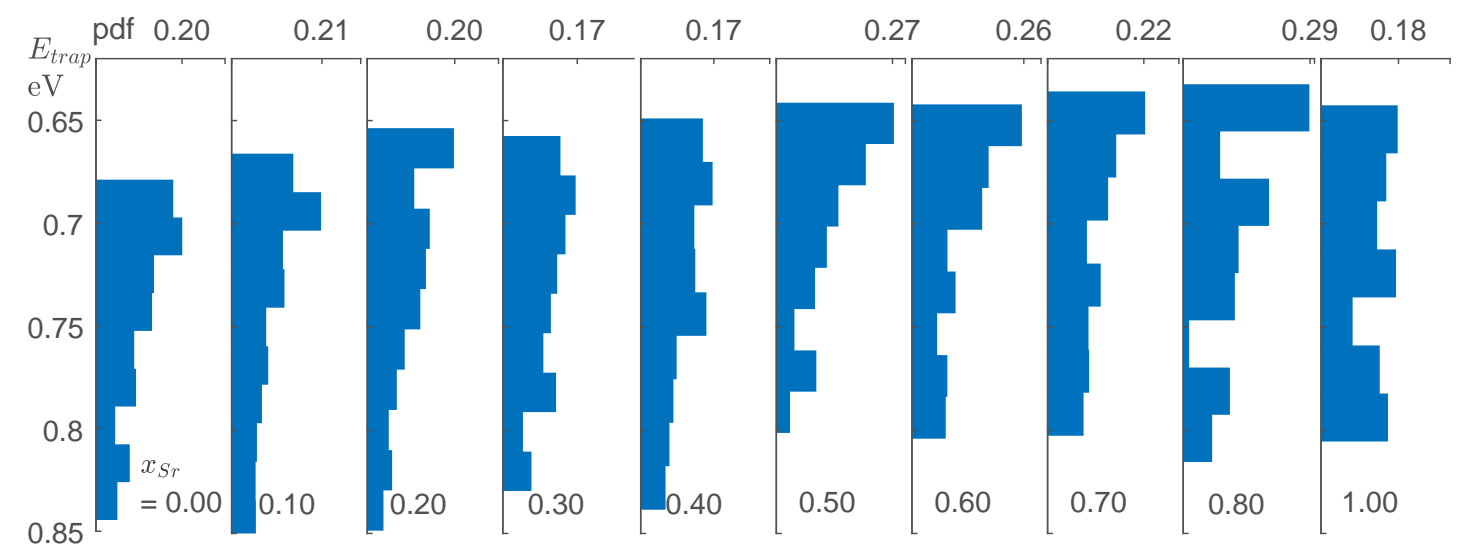

Figure 10: The normalized probability density function (pdf) of $n\left(E_{\text {trap }}, t\right)$ (at $t \simeq 0$ sec) in anorthite solid solutions for various $x_{S r}$. The $x_{S r}=0.3$ and 0.4 phosphors show very flat population. (All pdfs have been plotted in the range of $(0,0.30)$.)

maxima, which corresponds to the number of electrons in traps that are thermally accessible near room temperature. The magnitude of the TL peak maxima (Figure 9a and their corresponding total TL (integrated up to $190^{\circ} \mathrm{C}$ ) decrease with increasing $x_{S r}$ (Figure $9 \mathrm{~b}$ top), indicating the decrease of trapped electrons available in these phosphors. This explains the drop of the total afterglow intensity with $x_{S r}$. The trap depth $E_{\text {trap }}$ was estimated from the temperature of the TL peak maxima, $T_{m}$, according to the Urbach relation 68,

$$
E_{\text {trap }}(e V)=T_{m}(K) / c
$$

where $c$ is a constant that depends on the choice of $s$. In this case, we choose $s=10^{9} \mathrm{~Hz}$ and the constant $c$ is 500 68. Equation (8) only serves as an estimate of trap depth. The initial rise method provides the trap depth, regardless of the kinetics involved, but it requires high quality data in the initial rise region (up to $<15 \%$ of the TL peak maxima) 69 and it is not sensitive to small variation in $T_{m}$. Clearly, the trap depth first decreases till $x_{S r} \simeq 0.70$ and then increases slightly when $x_{S r}>0.7$ (Figure $9 \mathrm{~b}$ bottom). The variation is associated with the phase transition in anorthite solid solutions. Interestingly, the trap depth as a function of $x_{S r}$ when $x_{S r} \leq 0.70$ displays two regions where the slope is quite different, and the transition point is around $x_{S r} \simeq 0.30$, which has a slightly larger trap depth than expected. This may indicate the $\mathrm{P} \overline{1}-\mathrm{I} \overline{1}$ transition.

With increasing $x_{S r}$, the electron population function becomes broader in the ranges of $(0.0,0.4)$ and $(0.5,1.0)$ (Figure 10), separated by the $\mathrm{P} \overline{1}-\mathrm{I} \overline{1}$ transition. These broad distributions guarantee the correct asymptotic behavior of afterglow, i.e., $I(t) \sim t^{-\beta}$ with $\beta \simeq 1$. The electron population function $n\left(t, E_{\text {trap }}, q\right)$ at $E_{\text {trap }}$ depends on the time $t$ and initial charging conditions $q$. In the interval of trap depth $\left(E_{\text {trap }}, E_{\text {trap }}+d E\right)$, the number of electrons at time $t$ is,

$$
d n\left(t, E_{\text {trap }}, q\right)=N\left(E_{\text {trap }}\right) \cdot f\left(t, E_{\text {trap }}, q\right) \cdot d E
$$

where $N\left(E_{\text {trap }}\right)$ is the trap depth distribution and $f\left(t, E_{\text {trap }}, q\right)$ is the filling function $(0 \leq f<1)$ 70. The filling function is the ratio of the number of filled traps to the total number of traps at $E_{\text {trap }}$, and its value is limited due to low charging temperature, thermal detrapping, or even optically stimulated detrapping [71, 52. Thus, the trap depth distribution $N\left(E_{\text {trap }}\right)$, which can be evaluated according to the method in Ref 72 , does not provide enough information to determine $n\left(t, E_{\text {trap }}, q\right)$. Instead, the $T_{m}-T_{\text {stop }}$ method was adopted to meet the task 34] (section 3 in the ESI). A linear relationship between $T_{m}$ and $T_{\text {stop }}$ with a slope of $\simeq 1$ indicates the existence of a continuous distribution $n\left(t, E_{\text {trap }}, q\right)$ (Figure $\mathrm{S} 6$ in the ESI). These $T_{m}$ were converted into their corresponding $E_{\text {trap }}$ according to Equation (8), and the area between the TL curves of two neighboring $T_{m}$ was taken as a measure of electrons situated in the corresponding trap depth interval. Such a broad distribution for shallow-intermediate traps can already lead to Becquerel's decay law because its corresponding distribution of rate coefficient is a Gamma function 66 that encompasses both broad distribution (e.g., exponential distribution) and narrow distribution (e.g., Gaussian distribution), resulting in a decay function similar to Becquerel's decay law if the width of the distribution is broad enough 62.

The probability density functions (pdf) of $n\left(E_{\text {trap }}, t, q\right)$ for $x_{S r}=0.30$ and 0.40 stand out as they display appreciable states at intermediate-deep trap depth, resulting in very broad and flat distribution. This feature takes place in the $x_{S r}$ range where $\mathrm{P} \overline{1}-\mathrm{I} \overline{1}$ occurs. Interestingly, the pdf of $x_{S r}=0.7$ is broader than those of $x_{S r}=0.5$ or 0.6 . The larger $x_{S r}$, the broader the distribution in each range, due to the gradual influence of chemical substitution. For the investigated 1-700 sec Delay time, the pdfs change their shallow traps greatly, as the TL as a function of delay time after charging at $20{ }^{\circ} \mathrm{C}$ for $x_{S r}=0.0$ and 0.10 shows only a shift of $\sim 20 \mathrm{~K}$ in $T_{m}$, corresponding to a shift of trap depth of $\sim 0.04 \mathrm{eV}$ (see Figure S8 in the ESI). Given the low $\mathrm{ML}_{\text {Peak }}$ at large Delay time, very deep traps do 


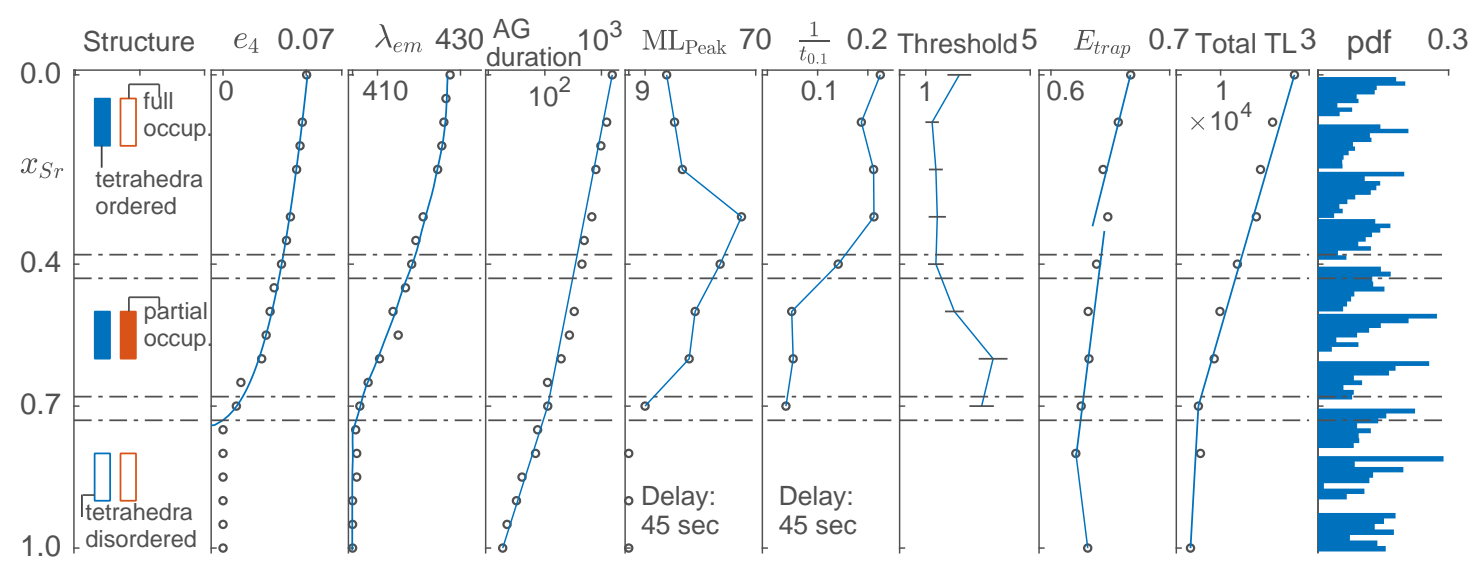

Figure 11: Both structural transitions, i.e., P $\overline{1}-\mathrm{I} \overline{1}$ and $\mathrm{I} \overline{1}-\mathrm{I} \frac{2}{c}$ transitions, are highly reflected in the ML properties and the electron population function. Meanwhile, the spontaneous strains, the PL $\lambda_{e m}$, the AG duration and the total TL show a change corresponding to the I $\overline{1}-\mathrm{I} \frac{2}{c}$ transition at $x_{S r} \in(0.70,0.75)$. Two pairs of dash-dotted grey lines indicate the possible range of phase transitions.

not contribute significantly to $\mathrm{ML}$ due to their relatively low density.

\subsection{The Structural Phase Transitions Are Reflected in Optical Properties}

Putting all the results together in Figure 11, we can easily see that the ML properties are very sensitive to the $\mathrm{P} \overline{1}-\mathrm{I} \overline{1}$ phase transition, which is hardly reflected in spontaneous strains, PL spectra (e.g. $\bar{\nu}_{e x}$ and $\lambda_{e m}$ ), AG duration or total TL. Interestingly, the pdf of electron population is quite flat when $x_{S r} \in(0.3,0.4)$. This shows strong evidence that the defect chemistry of the anorthites has an important impact on their optical properties.

It is not possible to ascribe ML properties to the structural defects directly, but certain speculations can be made at this moment. Normally, ML is believed to originate from the accelerated detrapping of electrons at traps due to the presence of an internal electric field near the defects during dynamic loading [73]. This process heavily depends on the symmetry and mechanical properties of the host of ML phosphors [74, as well as on the trap properties such as trap density and electron population function. Then, a disordered structure of cation polyhedra facilitates the detrapping of electrons because this disorder provides a wide range of states of electrons suitable for the detrapping due to the creation of the internal electric field, resulting in brighter ML. Such disorders also increase the rigidity of anorthite and thus produce higher IQE and ML threshold in the $\mathrm{I} \overline{1}$ phase. Moreover, ML is still selective to how the elastic properties couple with both trapping center and luminescent centers. In anorthites, ML is very sensitive to the I 1 - $\mathrm{I} \frac{2}{c}$ transition probably because of the symmetry of $\mathrm{I} \frac{2}{c}$ phase, while spontaneous strains, PL $\lambda_{e m}$, AG duration, and TL properties only show a gradual change near the transition point. Sensitive to both the symmetries of phosphor hosts and trap properties, ML seems quite suitable for the research of structural transitions in ML phosphors, especially near the transition point.

\section{Conclusion}

There are two important phase transitions in the anorthite solid solution $\mathrm{Ca}_{1-x} \mathrm{Sr}_{x} \mathrm{Al}_{2} \mathrm{Si}_{2} \mathrm{O}_{8}: \mathrm{Eu}^{2+}, \mathrm{Pr}^{3+}$, namely $\mathrm{P} \overline{1}-\mathrm{I} \overline{1}$ at $x_{S r} \simeq 0.40$ and $\mathrm{I} \overline{1}-\mathrm{I} \frac{2}{c}$ at $x_{S r} \simeq 0.70$. The occupation of $\mathrm{Al}^{3+} / \mathrm{Si}^{4+}$ at tetrahedral sites changes from ordered $\left(x_{S r} \in(0.0,0.70)\right)$ into disordered $\left(x_{S r} \in(0.70,1.00)\right)$, while the cation sites are partially occupied by $\mathrm{Ca}^{2+} / \mathrm{Sr}^{2+}$ (atom-split) only in the range of $x_{S r} \in(0.4,0.70)$. The İ1-I $\frac{2}{c}$ transition can be well deduced from spontaneous strains, PL spectra, AG duration, total TL intensities and also the presence of ML intensities. Meanwhile, the P $\overline{1}-\mathrm{I} \overline{1}$ transition can only be reflected in the evolution of IQE, ML intensities, the ML thresholds, and average decay rate of ML tail $\frac{1}{t_{0.1}}$, as these quantities show a transition when $x_{S r} \simeq 0.4$. ML $L_{\text {Peak }}$ and $\frac{1}{t_{0.1}}$ follow a power law decay with Delay time because of the accelerated detrapping of electrons from traps. In addition, the electron population functions are broad distributions, which leads to the correct afterglow profiles, and are sensitive to reveal both transitions. Sensitive to the coupling between elasticity (and the symmetries) of the host and the local structures or/and trap properties, ML may serve as a very sensitive probe that allows to investigate structural transitions in ML phosphors. It extends our toolbox to investigate properties of ML phosphors.

\section{Acknowledgment}

This research was supported by the Special Research Fund (BOF) of Ghent University through the GOA project "Enclose". Simon Michels acknowledges the financial support of FWO-Vlaanderen (SB grant 1S33317N). We thank Olivier Janssens for his assistance in measuring XRD of the samples. 


\section{References}

[1] A. Feng, P. F. Smet, A review of mechanoluminescence in inorganic solids: Compounds, mechanisms, models and applications, Materials 11 (4) (2018) 484. doi:10.3390/ma11040484

[2] C.-N. Xu, T. Watanabe, M. Akiyama, X.-G. Zheng, Direct view of stress distribution in solid by mechanoluminescence, Appl. Phys. Lett. 74 (17) (1999) 2414-2416. doi:10.1063/1.123865

[3] S. M. Jeong, S. Song, K.-I. Joo, J. Kim, S.-H. Hwang, J. Jeong, H. Kim, Bright, wind-driven white mechanoluminescence from zinc sulphide microparticles embedded in a polydimethylsiloxane elastomer, Energy Environ. Sci. 7 (10) (2014) 3338-3346. doi:10.1039/C4EE01776E

[4] S. Song, B. Song, C.-H. Cho, S. K. Lim, S. M. Jeong, Textilefiber-embedded multiluminescent devices: A new approach to soft display systems, Mater. Todaydoi:10.1016/j.mattod. 2019.08.004

[5] S. M. Jeong, S. Song, H.-J. Seo, W. M. Choi, S.-H. Hwang, S. G. Lee, S. K. Lim, Battery-free, human-motion-powered lightemitting fabric: Mechanoluminescent textile, Adv. Sustainable Syst. 1 (12) (2017) 1700126. doi:10.1002/adsu.201700126

[6] X. Qian, Z. Cai, M. Su, F. Li, W. Fang, Y. Li, X. Zhou, Q. Li, X. Feng, W. Li, X. Hu, X. Wang, C. Pan, Y. Song, Printable skin-driven mechanoluminescence devices via nanodoped matrix modification, Adv. Mater. 30 (25) (2018) 1800291. doi:10. 1002/adma.201800291

[7] Z. Ma, J. Zhou, J. Zhang, S. Zeng, H. Zhou, A. T. Smith, W. Wang, L. Sun, Z. Wang, Mechanics-induced triple-mode anticounterfeiting and moving tactile sensing by simultaneously utilizing instantaneous and persistent mechanoluminescence, Mater. Horiz doi:10.1039/C9MH01028A.

[8] J.-C. Zhang, C. Pan, Y.-F. Zhu, L.-Z. Zhao, H.-W. He, X. Liu, J. Qiu, Achieving thermo-mechano-opto-responsive bitemporal colorful luminescence via multiplexing of dual lanthanides in piezoelectric particles and its multidimensional anticounterfeiting, Adv. Mater. 30 (49) (2018) 1804644. doi:10.1002/adma. 201804644

[9] L. Chen, M.-C. Wong, G. Bai, W. Jie, J. Hao, White and green light emissions of flexible polymer composites under electric field and multiple strains, Nano Energy 14 (2015) 372-381. doi: 10.1016/j.nanoen.2014.11.039

[10] M.-C. Wong, L. Chen, G. Bai, L.-B. Huang, J. Hao, Temporal and remote tuning of piezophotonic-effect-induced luminescence and color gamut via modulating magnetic field, Adv. Mater. 29 (43) (2017) 1701945. doi:10.1002/adma. 201701945

[11] X. Wang, M. Que, M. Chen, X. Han, X. Li, C. Pan, Z. L. Wang, Full dynamic-range pressure sensor matrix based on optical and electrical dual-mode sensing, Adv. Mater. 29 (15) (2017) 1605817. doi:10.1002/adma.201605817

[12] K.-S. Sohn, S. Timilsina, S. P. Singh, T. Choi, J. S. Kim, Mechanically driven luminescence in a $\mathrm{ZnS}: \mathrm{Cu}-\mathrm{PDMS}$ composite, APL Mater. 4 (10) (2016) 106102. doi:10.1063/1.4964139

[13] S. I. Bredikhin, S. Z. Shmurak, Deformation-stimulated emission of ZnS crystal, JETP Lett. 19 (12) (1974) 367-368.

[14] J.-C. Zhang, X. Wang, G. Marriott, C.-N. Xu, Trap-controlled mechanoluminescent materials, Prog. Mater Sci. 103 (2019) 678-742. doi:10.1016/j.pmatsci.2019.02.001

[15] X. Wang, C.-N. Xu, H. Yamada, K. Nishikubo, X.-G. Zheng, Electro-Mechano-Optical conversions in $\mathrm{Pr}^{3+}$-coped $\mathrm{BaTiO}_{3}$ $\mathrm{CaTiO}_{3}$ ceramics, Adv. Mater. 17 (10) (2005) 1254-1258. doi : 10.1002/adma.200401406

[16] X. Wang, H. Yamada, C.-N. Xu, Large electrostriction near the solubility limit in $\mathrm{BaTiO}_{3}-\mathrm{CaTiO}_{3}$ ceramics, Appl. Phys. Lett. 86 (2) (2005) 022905. doi:10.1063/1.1850598

[17] J.-C. Zhang, X.-H. Fan, X. Yan, F. Xia, W. Kong, Y.-Z. Long, $\mathrm{X}$. Wang, Sacrificing trap density to achieve short-delay and high-contrast mechanoluminescence for stress imaging, Acta Mater. 152 (2018) 148-154. doi:10.1016/j.actamat.2018.04. 011

[18] N. Goldenfeld, Lectures on Phase Transitions and the Renormalization Group, no. Vol. 85 in Frontiers in Physics, CRC Press, Taylor \& Francis Group, USA ; Boca Raton, F.L., 1992.
[19] M. A. Carpenter, C. J. Howard, M. J. Andrew, R. E. A. McKnight, Y. Liu, R. L. Withers, Elastic anomalies due to structural phase transitions in mechanoluminescent $\mathrm{SrAl}_{2} \mathrm{O}_{4}: \mathrm{Eu}$, J. Appl. Phys. 107 (1) (2010) 013505. doi:10.1063/1.3269723

[20] T. Takenaka, H. Nagata, Y. Hiruma, Phase transition temperatures and piezoelectric properties of $\left(\mathrm{Bi}_{1 / 2} \mathrm{Na}_{1 / 2}\right) \mathrm{TiO}_{3}$-and $\left(\mathrm{Bi}_{1 / 2} \mathrm{~K}_{1 / 2}\right) \mathrm{TiO}_{3}$-based bismuth perovskite lead-free ferroelectric ceramics, IEEE Trans. Ultrason., Ferroelectr., Freq. Control 56 (8) (2009) 1595-1612. doi:10.1109/TUFFC. 2009.1224.

[21] F. Li, D. Lin, Z. Chen, Z. Cheng, J. Wang, C. Li, Z. Xu, Q. Huang, X. Liao, L.-Q. Chen, T. R. Shrout, S. Zhang, Ultrahigh piezoelectricity in ferroelectric ceramics by design, Nat. Mater. 17 (4) (2018) 349-354. doi:10.1038/ s41563-018-0034-4

[22] J. Remeika, A. Glass, The growth and ferroelectric properties of high resistivity single crystals of lead titanate, Mater. Res. Bull. 5 (1) (1970) 37-45. doi:10.1016/0025-5408(70)90071-1

[23] H. D. Megaw, C. J. E. Kempster, E. W. Radoslovich, The structure of anorthite, $\mathrm{CaAl}_{2} \mathrm{Si}_{2} \mathrm{O}_{8}$.II. Description and discussion, Acta Crystallogr. 15 (10) (1962) 1017-1035. doi:10.1107/ S0365110X62002637

[24] S. Ghose, G. Van Tendeloo, S. Amelinckx, Dynamics of a second-order phase transition: $\mathrm{P} \overline{1}$ to $\mathrm{I} \overline{1}$ phase transition in anorthite, $\mathrm{CaAl}_{2} \mathrm{Si}_{2} \mathrm{O}_{8}$, Science 242 (4885) (1988) 1539-1541. doi:10.1126/science.242.4885.1539

[25] M. D. McGuinn, S. A. T. Redfern, Ferroelastic phase transition in $\mathrm{SrAl}_{2} \mathrm{Si}_{2} \mathrm{O}_{8}$ feldspar at elevated pressure, Mineral. Mag. 58 (390) (1994) 21-26. doi:10.1180/minmag.1994.058.390.02.

[26] M. D. McGuinn, S. A. T. Redfern, Ferroelastic phase transition along the join $\mathrm{CaAl}_{2} \mathrm{Si}_{2} \mathrm{O}_{8}-\mathrm{SrAl}_{2} \mathrm{Si}_{2} \mathrm{O}_{8}, \mathrm{Am}$. Mineral. 79 (1-2) (1994) 24-30. doi:10.2138/am-1994-1-024

[27] R. J. Angel, N. L. Ross, J. Zhao, L. Sochalski-Kolbus, H. Krüger, B. C. Schmidt, Structural controls on the anisotropy of tetrahedral frameworks: the example of monoclinic feldspars, Eur. J. Mineral. 25 (4) (2013) 597-614. doi:10.1127/ 0935-1221/2013/0025-2323

[28] J.-C. Zhang, Y.-Z. Long, X. Yan, X. Wang, F. Wang, Creating recoverable mechanoluminescence in piezoelectric calcium niobates through $\mathrm{Pr}^{3+}$ doping, Chem. Mater. 28 (11) (2016) 4052-4057. doi:10.1021/acs.chemmater.6b01550

[29] L. Zhang, C.-N. Xu, H. Yamada, N. Bu, Enhancement of mechanoluminescence in $\mathrm{CaAl}_{2} \mathrm{Si}_{2} \mathrm{O}_{8}: \mathrm{Eu}^{2+}$ by partial $\mathrm{Sr}^{2+}$ substitution for $\mathrm{Ca}^{2+}$, J. Electrochem. Soc. 157 (3) (2010) J50. doi:10.1149/1.3274879

[30] H. Fang, G. Qiu, J. Li, X. Wang, $\mathrm{Sr}^{2+}$ substitution for $\mathrm{Ca}^{2+}$ and $\mathrm{Eu}^{2+}, \mathrm{Dy}^{3+}$ co-doping enhance mechanoluminescence of $\mathrm{CaAl}_{2} \mathrm{Si}_{2} \mathrm{O}_{8}$ phosphors, J. Alloys Compd. 763 (2018) 267-272. doi:10.1016/j.jallcom.2018.05.294

[31] J. Rodríguez-Carvajal, Recent advances in magnetic structure determination by neutron powder diffraction, Physica B 192 (1) (1993) 55-69. doi:10.1016/0921-4526(93)90108-I

[32] F. Menges, Spectragryph - optical spectroscopy software, [Online; accessed 2019-10-02]. URL https://www .effemm2.de/spectragryph/

[33] J. Botterman, J. J. Joos, P. F. Smet, Trapping and detrapping in $\mathrm{SrlAl}_{2} \mathrm{O}_{4}: \mathrm{Eu}, \mathrm{Dy}$ persistent phosphors: Influence of excitation wavelength and temperature, Phys. Rev. B: Condens. Matter 90 (8) (2014) 085147. doi:10.1103/PhysRevB.90.085147.

[34] S. W. S. McKeever, On the analysis of complex thermoluminescence glow-curves: Resolution into individual peaks, Phys. Status Solidi A 62 (1) (1980) 331-340. doi:10.1002/pssa. 2210620139

[35] A. Feng, S. Michels, A. Lamberti, P. F. Smet, Mechanoluminescent materials: A new way to analyze stress by light, Proceedings 2 (8) (2018) 492. doi:10.3390/ICEM18-05422

[36] M. Tribaudino, P. Benna, F. Nestola, C. Meneghini, E. Bruno, Thermodynamic behaviour of the high-temperature $\mathrm{P} \overline{1}-\mathrm{I} \overline{1}$ phase transition along the $\mathrm{CaAl}_{2} \mathrm{Si}_{2} \mathrm{O}_{8}-\mathrm{SrAl}_{2} \mathrm{Si}_{2} \mathrm{O}_{8}$ join, Phys. Chem. Miner. 32 (4) (2005) 314-321. doi:10.1007/ s00269-005-0469-4

[37] S. Ghose, R. K. McMullan, H.-P. Weber, Neutron diffraction 
studies of the $\mathrm{P} \overline{1}-\mathrm{I} \overline{1}$ transition in anorthite, $\mathrm{CaAl}_{2} \mathrm{Si}_{2} \mathrm{O}_{8}$, and the crystal structure of the body-centered phase at $514 \mathrm{~K}, \mathrm{Z}$. Kristallogr. Cryst. Mater. 204 (1-2) (1993) 215-237. doi:10. 1524/zkri.1993.204.12.215

[38] R. J. Angel, L. M. Sochalski-Kolbus, M. Tribaudino, Tilts and tetrahedra: The origin of the anisotropy of feldspars, Am. Mineral. 97 (5-6) (2012) 765-778. doi:10.2138/am.2012.4011

[39] K. Momma, F. Izumi, VESTA3 for three-dimensional visualization of crystal, volumetric and morphology data, Journal of Applied Crystallography 44 (6) (2011) 1272-1276. doi: 10.1107/S0021889811038970

40] M. A. Carpenter, E. K. Salje, A. Graemer-Barber, Spontaneous strain as a determinant of thermodynamic properties for phase transions in minerals, Eur. J. Mineral. 10 (4) (1998) 621-691.

[41] M. Tribaudino, M. Zhang, E. K. H. Salje, Cation ordering and phase transitions in feldspars along the join $\mathrm{CaAl}_{2} \mathrm{Si}_{2} \mathrm{O}_{8}-\mathrm{SrAl}_{2} \mathrm{Si}_{2} \mathrm{O}_{8}$ : A TEM, IR and XRD investigation, Mineral. Mag. 73 (01) (2009) 119-130. doi:10.1180/minmag. 2009.073.1.119

[42] M. T. Dove, S. A. T. Redfern, Lattice simulation studies of the ferroelastic phase transitions in $(\mathrm{Na}, \mathrm{K}) \mathrm{AlSi}_{3} \mathrm{O}_{8}$ and ( $\mathrm{Sr}, \mathrm{Ca}) \mathrm{Al}_{2} \mathrm{Si}_{2} \mathrm{O}_{8}$ feldspar solid solutions, Am. Mineral. 82 (1-2) (1997) 8-15. doi:10.2138/am-1997-1-202

[43] M. Tribaudino, P. Benna, E. Bruno, I1- $\frac{2}{2}$ phase transition in alkaline-earth feldspars along the $\mathrm{CaAl}_{2} \mathrm{Si}_{2} \mathrm{O}_{8}-\mathrm{SrAl}_{2} \mathrm{Si}_{2} \mathrm{O}_{8}$ join: Thermodynamic behaviour, Phys. Chem. Miner. 20 (4) (1993) 221-227. doi:10.1007/BF00208135

$44]$ Z. Wang, Y. Wang, P. Zhang, X. Fan, G. Qian, Tunable afterglow color in $\mathrm{Eu}^{2+}$ and $\mathrm{Dy}^{3+}$ co-activated alkaline earth feldspar solid solutions phosphors, J. Lumin. 124 (1) (2007) 140 - 142. doi:10.1016/j.jlumin.2006.02.015

[45] R. D. Shannon, Revised effective ionic radii and systematic studies of interatomic distances in halides and chalcogenides, Acta Crystallogr., Sect. A 32 (5) (1976) 751-767. doi:10.1107/ S0567739476001551

[46] A. Allred, Electronegativity values from thermochemical data, J. Inorg. Nucl. Chem. 17 (3) (1961) 215 - 221. doi:10.1016/ 0022-1902(61)80142-5.

[47] P. Dorenbos, Crystal field splitting of lanthanide $4 \mathrm{f}^{n-1} 5 \mathrm{~d}$-levels in inorganic compounds, J. Alloys Compd. 341 (1) (2002) 156159. doi:10.1016/S0925-8388(02) 00056-7

[48] P. Dorenbos, $5 d$-level energies of $\mathrm{Ce}^{3+}$ and the crystalline environment. i. fluoride compounds, Phys. Rev. B 62 (2000) 1564015649. doi:10.1103/PhysRevB.62.15640

[49] P. Dorenbos, $5 d$ - level energies of $\mathrm{Ce}^{3+}$ and the crystalline environment. ii. chloride, bromide, and iodide compounds, Phys. Rev. B 62 (2000) 15650-15659. doi:10.1103/PhysRevB.62. 15650

[50] P. Dorenbos, $5 d$-level energies of $\mathrm{Ce}^{3+}$ and the crystalline environment. iii. oxides containing ionic complexes, Phys. Rev. B 64 (2001) 125117. doi:10.1103/PhysRevB.64.125117

[51] L. He, Z. Song, X. Jia, Z. Xia, Q. Liu, Consequence of optimal bonding on disordered structure and improved luminescence properties in t-phase $(\mathrm{Ba}, \mathrm{Ca})_{2} \mathrm{SiO}_{4}: \mathrm{Eu}^{2+}$ phosphor, Inorg. Chem. 57 (7) (2018) 4146-4154. doi:10.1021/acs. inorgchem.8b00362

[52] D. Van der Heggen, J. J. Joos, P. F. Smet, Importance of evaluating the intensity dependency of the quantum efficiency: Impact on leds and persistent phosphors, ACS Photonics 5 (11) (2018) 4529-4537. doi:10.1021/acsphotonics.8b00979

[53] X. Yu, X. Xu, H. Yu, T. Jiang, P. Yang, Q. Jiao, J. Qiu, Persistence energy transfer between inequivalent $\mathrm{Eu}^{2+}$ ions in $\mathrm{CaAl}_{2} \mathrm{Si}_{2} \mathrm{O}_{8}: \mathrm{Eu}^{2+}$, Mater. Res. Bull. 47 (9) (2012) $2696-2699$. doi:10.1016/j.materresbull.2012.04.024

[54] M. E. Becquerel, La lumière, ses causes et ses effets, Librairie de Firmin Didot Frères, Paris, 1867.

[55] K. Van den Eeckhout, P. F. Smet, D. Poelman, Persistent luminescence in $\mathrm{Eu}^{2+}$-doped compounds: A review, Materials 3 (4) (2010) 2536-2566. doi:10.3390/ma3042536

[56] R. Chen, S. W. McKeever, Thermoluminescence (TL), World Scientific, Farrer Road, Singapore, 1997.
[57] E. Adirovitch, La formule de Becquerel et la loi élémentaire $\mathrm{du}$ déclin de la luminescence des phosphores cristallins, J. Phys. Radium 17 (8-9) (1956) 705-707. doi:10.1051/jphysrad: 01956001708-9070500

[58] R. T. Ellickson, W. L. Parker, Decay in brightness of infrared sensitive phosphors, Phys. Rev. 70 (1946) 290-299. doi: 10.1103/PhysRev.70.290

59] C. M. Sunta, The Quasi-Equilibrium Problem in Thermoluminescence, Springer India, New Delhi, 2015. doi:10.1007/ 978-81-322-1940-8_5

[60] W. F. Hornyak, R. Chen, Thermoluminescence and phosphorescence with a continuous distribution of activation energies, J. Lumin. 44 (1) (1989) 73-81. doi:10.1016/0022-2313(89) 90023-9

[61] M. N. Berberan-Santos, B. Valeur, Luminescence decays with underlying distributions: General properties and analysis with mathematical functions, J. Lumin. 126 (2) (2007) 263-272. doi : 10.1016/j.jlumin.2006.07.004.

[62] W. L. Medlin, Decay of phosphorescence from a distribution of trapping levels, Phys. Rev. 123 (1961) 502-509. doi:10.1103/ PhysRev.123.502

[63] J. T. Randall, M. H. F. Wilkins, Phosphorescence and electron traps II. The interpretation of long-period phosphorescence, Proc. R. Soc. Lond. A 184 (999) (1945) 390-407. doi: 10.1098/rspa.1945.0025

64] M. Berberan-Santos, E. Bodunov, B. Valeur, Mathematical functions for the analysis of luminescence decays with underlying distributions: 2. becquerel (compressed hyperbola) and related decay functions, Chem. Phys. 317 (1) (2005) $57-62$. doi:10.1016/j.chemphys.2005.05.026

[65] M. Tribaudino, P. Benna, E. Bruno, TEM observations on the $\mathrm{P} \overline{1}-\mathrm{I} \overline{1}$ phase transition in feldspars along the join $\mathrm{CaAl}_{2} \mathrm{Si}_{2} \mathrm{O}_{8}-$ $\mathrm{SrAl}_{2} \mathrm{Si}_{2} \mathrm{O}_{8}$, Am. Mineral. 85 (7-8) (200) 963-970. doi:10. 2138/am-2000-0710.

[66] L. Whitehead, R. Whitehead, B. Valeur, M. Berberan-Santos, A simple function for the description of near-exponential decays: The stretched or compressed hyperbola, Am. J. Phys. 77 (2) (2009) 173-179. doi:10.1119/1.3008007

[67] M. N. Berberan-Santos, E. N. Bodunov, B. Valeur, Luminescence decays with underlying distributions of rate constants: General properties and selected cases, in: M. N. BerberanSantos (Ed.), Fluorescence of Supermolecules, Polymers, and Nanosystems, Vol. 4, Springer Berlin Heidelberg, Berlin, Heidelberg, 2007, pp. 67-103. doi:10.1007/4243_2007_001

[68] F. Urbach, Zur Lumineszenz der Alkalihalogenide, Sitzungsberichte Akad. der Wiss. Wien 139 (1930) 363-372.

[69] G. F. J. Garlick, A. F. Gibson, The electron trap mechanism of luminescence in sulphide and silicate phosphors, Proc. Phys. Soc. 60 (6) (1948) 574. doi:10.1088/0959-5309/60/6/308

[70] R. Chen, S. W. S. McKeever, Theory of thermoluminescence and related phenomena, World Scientific, Singapore ; River Edge, N.J, 1997.

[71] C. Tydtgat, K. W. Meert, D. Poelman, P. F. Smet, Optically stimulated detrapping during charging of persistent phosphors, Opt. Mater. Express 6 (3) (2016) 844. doi:10.1364/0ME.6. 000844

[72] K. Van den Eeckhout, A. J. J. Bos, D. Poelman, P. F. Smet, Revealing trap depth distributions in persistent phosphors, Phys. Rev. B 87 (4) (2013) 045126. doi:10.1103/PhysRevB. 87.045126

[73] V. Chandra, B. Chandra, P. Jha, Models for intrinsic and extrinsic elastico and plastico-mechanoluminescence of solids, J. Lumin. 138 (2013) 267-280. doi:10.1016/j.jlumin.2013.01. 024

[74] J. A. Sussmann, Electric dipoles due to trapped electrons, Proc. Phys. Soc. 79 (4). doi:10.1088/0370-1328/79/4/313 